Review

\title{
Multi-Beam Interference Advances and Applications: Nano-Electronics, Photonic Crystals, Metamaterials, Subwavelength Structures, Optical Trapping, and Biomedical Structures
}

\section{Guy M. Burrow and Thomas K. Gaylord *}

School of Electrical and Computer Engineering, Georgia Institute of Technology, Atlanta, GA 30332, USA; E-Mail: gburrow3@mail.gatech.edu

* Author to whom correspondence should be addressed; E-Mail: tgaylord@ece.gatech.edu; Tel.: +1-404-894-2931; Fax: +1-404-894-2979.

Received: 14 April 2011; in revised form: 25 May 2011 / Accepted: 27 May 2011 / Published: 3 June 2011

\begin{abstract}
Research in recent years has greatly advanced the understanding and capabilities of multi-beam interference (MBI). With this technology it is now possible to generate a wide range of one-, two-, and three-dimensional periodic optical-intensity distributions at the micro- and nano-scale over a large length/area/volume. These patterns may be used directly or recorded in photo-sensitive materials using multi-beam interference lithography (MBIL) to accomplish subwavelength patterning. Advances in MBI and MBIL and a very wide range of applications areas including nano-electronics, photonic crystals, metamaterials, subwavelength structures, optical trapping, and biomedical structures are reviewed and put into a unified perspective.
\end{abstract}

Keywords: multi-beam interference; interference lithography; nano-electronics; photonic crystals; metamaterials; subwavelength structures; optical trapping; biomedical structures

\section{Introduction}

Nothing has had a greater impact on our daily lives than microelectronics. The semiconductor revolution is the engine that drives cell phones, the internet, flat-panel televisions, flash memory chips, global positioning system devices, solar cells, etc. Microelectronics has had a profound impact on the fields of biomedicine, transportation, communications, entertainment, defense, environmental monitoring, 
and homeland security [1]. In 1965, Gordon Moore, a co-founder of Intel, predicted that the number of transistors in a commercial integrated circuit would double every two years. For more than three decades optical lithography has been the enabling technology to meet the semiconductor industry's push to decrease device size and satisfy Moore's law. However, today there is serious doubt that conventional optical lithography can continue to provide the needed decreasing sizes. Efforts to reduce the wavelength of the source (e.g., from $192 \mathrm{~nm}$ to $157 \mathrm{~nm}$ ) have not met commercial success. Increasing the surrounding refractive index in immersion lithography (e.g., from 1.44 to 1.65) remains a research and development tool. New approaches are needed. Techniques being considered include (1) self-assembly approaches; (2) construction-based approaches including immersion lithography, double patterning, two-photon lithography, printing, direct writing, mask optimization, and micromanipulation; and, (3) interference lithography [2-5].

Among these approaches, multi-beam interference lithography (MBIL), sometimes referred to in the literature as "holographic" or "interferometric" lithography, has emerged as a promising technology for relatively simple, subwavelength, and cost-effective periodic patterning in one, two and even three dimensions. Over the past decade, research has provided a comprehensive understanding of multi-beam interference (MBI) patterning possibilities and optimization requirements. Naturally, much effort has focused on the use of MBIL to meet micro- and nano-electronic fabrication requirements. However, MBI has found numerous additional applications outside the microelectronics industry, specifically in the fields of photonic crystals, metamaterials, subwavelength structures, optical trapping, and biomedical structures. Several review papers are available that describe MBI methods and capabilities [3,6,7] while a recent review focuses on the impact of interference lithography on nanostructure research [8]. The present work complements these papers, providing a review of the advances in MBI and MBIL and their use in nano-electronics, followed by a unified, comprehensive discussion of other current applications.

\section{Multi-Beam Interference}

An understanding of MBI may be developed by first considering the familiar interference pattern produced by two linearly-polarized, monochromatic, plane waves derived from a common coherent source. Each plane wave may be defined by $E_{i}(r, t)=E_{i} \cos \left(\omega t-k_{i} \cdot r+\phi_{i}\right) \hat{e}_{i}$, where $E_{i}$ is the amplitude, $\omega$ is the frequency, $\mathbf{k}_{i}$ is the wavevector, $\phi_{\mathrm{i}}$ is the phase, and $\hat{e}_{i}$ represents the linear polarization vector, and $i=1,2$. The interference of the two plane waves produces a one-dimensional (1D) fringe pattern with a periodicity $\Lambda$ directly proportional to the wavelength $\lambda$ and inversely proportional to the sine of the sum of the wavevector incidence angles $\theta$ with respect to the $z$ axis as depicted in Figure 1.

The total time-independent intensity distribution $I_{T}(\mathrm{r})$ at the intersection of the two waves is expressed as the absolute square of the sum of the amplitudes $I_{T}(r)=\left|E_{1}(r)+E_{2}(r)\right|^{2}$. For the wavevector configuration depicted in Figure 1, the total intensity distribution may be given by

$$
I_{T}(r)=I_{0}\left[1+\frac{E_{1} E_{2}\left(\hat{e}_{1} \cdot \hat{e}_{2}\right)}{I_{0}} \cos \left(k_{0} 2 \sin \theta \hat{x}+\Phi_{1}-\Phi_{2}\right)\right]
$$


where $k_{0}=2 \pi / \lambda$ and a DC intensity term $I_{0}=\frac{1}{2}\left(E_{1}^{2}+E_{2}^{2}\right)$ is introduced. From Equation 1 , we see that the spatial-cosine term describes a $1 \mathrm{D}$ periodic pattern that varies along the $x$ axis with a periodicity of $\Lambda=\lambda / 2 \sin \theta[9]$ as depicted in Figure 1 .

Figure 1. Two-beam interference. Interference fringes at the $x-y$ plane with a periodicity of $\Lambda$ are formed by two linearly-polarized, monochromatic, plane waves. In this example, $\mathbf{k}_{I}$ and $\mathbf{k}_{2}$ are contained in the $y-z$ plane at an angle $\theta$ with respect to the $z$ axis.

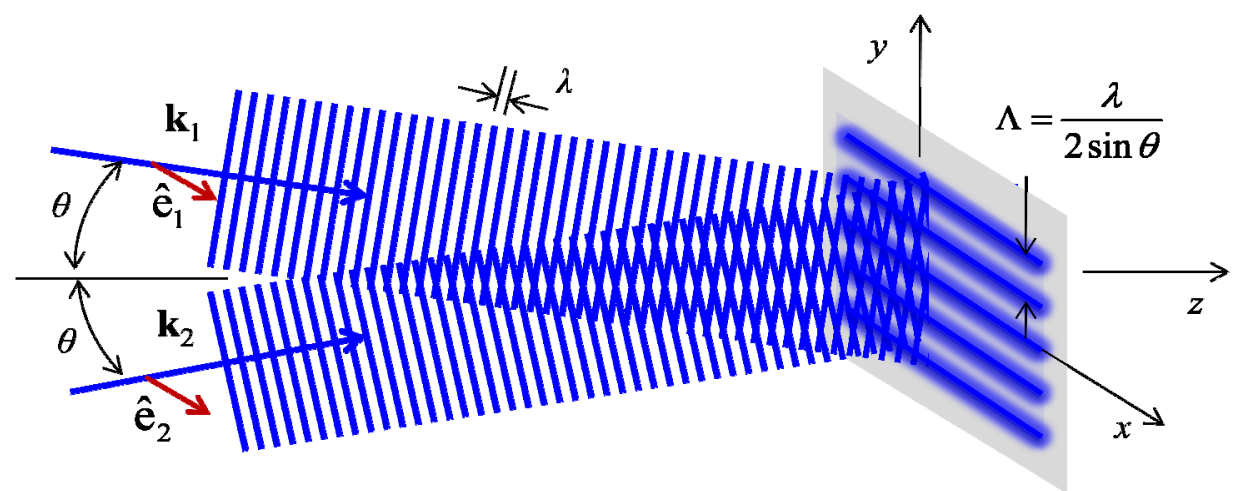

For $N$ interfering beams, the general form of the total time-independent intensity distribution $I_{T}(\mathrm{r})$ may given as

$$
I_{T}(r)=I_{0}\left[1+\sum_{j>i}^{N} V_{i j} \cos \left(\left(k_{j}-k_{i}\right) \cdot r+\Phi_{i}-\Phi_{j}\right)\right]
$$

where $I_{o}$ is now $\frac{1}{2} \sum_{k=1}^{N} E_{k}^{2}$ and an interference coefficient $V_{i j}$ is introduced and defined as

$$
V_{i j}=\frac{E_{i} E_{j}\left(e_{i} \cdot e_{j}\right)}{I_{0}}
$$

In Equation 2, we see that every beam-pair combination produces a fringe pattern, each defined by a spatial-cosine term with magnitude $V_{i j}$ that contributes to the overall periodic optical-intensity distribution. The orientation and periodicity of these $1 \mathrm{D}$ fringe patterns are determined by the relative beam-pair wavevector configurations and angles of incidence, while the relative phase difference determines the position of the fringes along the dimensional axis. In general, three-beam interference produces up to three 1D fringe patterns that combine to form a two-dimensional (2D) pattern as depicted in Figure 2. Four-beam interference provides three-dimensional (3D) patterning with up to six interfering beam pairs. Increasingly complex designs are realized as the number of interfering beams increases and elliptical or circular beam polarizations are considered. Together, the configuration of the wavevectors determines the translational symmetry of the periodic pattern.

The geometry of the unit cell within the periodic pattern further defines the space group symmetry and is determined by the amplitude and polarization of the individual beams. Specifically, the magnitude of each interference coefficient, as defined by Equation 3, is proportional to the product of the amplitudes $E_{i} E_{j}$ and the dot product of the polarization vectors $e_{i} \cdot e_{j}$ for each beam pair. The interference coefficient for each spatial cosine term, in turn, determines the contrast and relative contribution of each beam-pair fringe pattern to the overall periodic distribution. The significance of 
the individual beam polarizations is demonstrated in Figure 2. By adjusting the relative linear polarizations of the beams defined by $\mathbf{k}_{2}$ and $\mathbf{k}_{3}$, the interference coefficient $V_{23}$ may be set to a zero value when the two polarization vectors are mutually orthogonal, thereby eliminating the fringe pattern of Figure 2(d) altogether. In this case, assuming equal contrast for the two remaining fringe patterns ( $\left.V_{12}=V_{13}\right)$, a square lattice is formed with $p 4 m$ plane group symmetry as depicted in Figure 2(e). Alternatively, for the same wavevector configuration, the relative polarizations may be selected such that all three interference coefficients are equal ( $\left.V_{12}=V_{13}=V_{23}\right)$, again combining to form a square lattice, but now with $\mathrm{cmm}$ plane group symmetry as depicted in Figure 2(f).

Figure 2. Three-beam interference. (a) Three beams defined by $\mathbf{k}_{1}, \mathbf{k}_{2}$, and $\mathbf{k}_{3}$ interfere at the $x-y$ plane at a common angle of incidence $\theta$ with respect to the $z$ axis. The beam pairs defined by (b) $\mathbf{k}_{1}$ and $\mathbf{k}_{2}$, (c) $\mathbf{k}_{1}$ and $\mathbf{k}_{3}$, and (d) $\mathbf{k}_{2}$ and $\mathbf{k}_{3}$ form three distinct 1D-interference fringe patterns. (e) The fringes patterns of $\mathbf{k}_{1}$ and $\mathbf{k}_{2}$ and $\mathbf{k}_{1}$ and $\mathbf{k}_{3}$ combine to form a square lattice with $p 4 m$ plane group symmetry. (f) The fringe pattern of $\mathbf{k}_{2}$ and $\mathbf{k}_{3}$ combine with the other two fringe patterns to form a square lattice with $\mathrm{cmm}$ plane group symmetry. The lattice constant $a$ for the resulting pattern is directly proportional to the common beam wavelength $\lambda$ and inversely proportional to the sine of $\theta$.

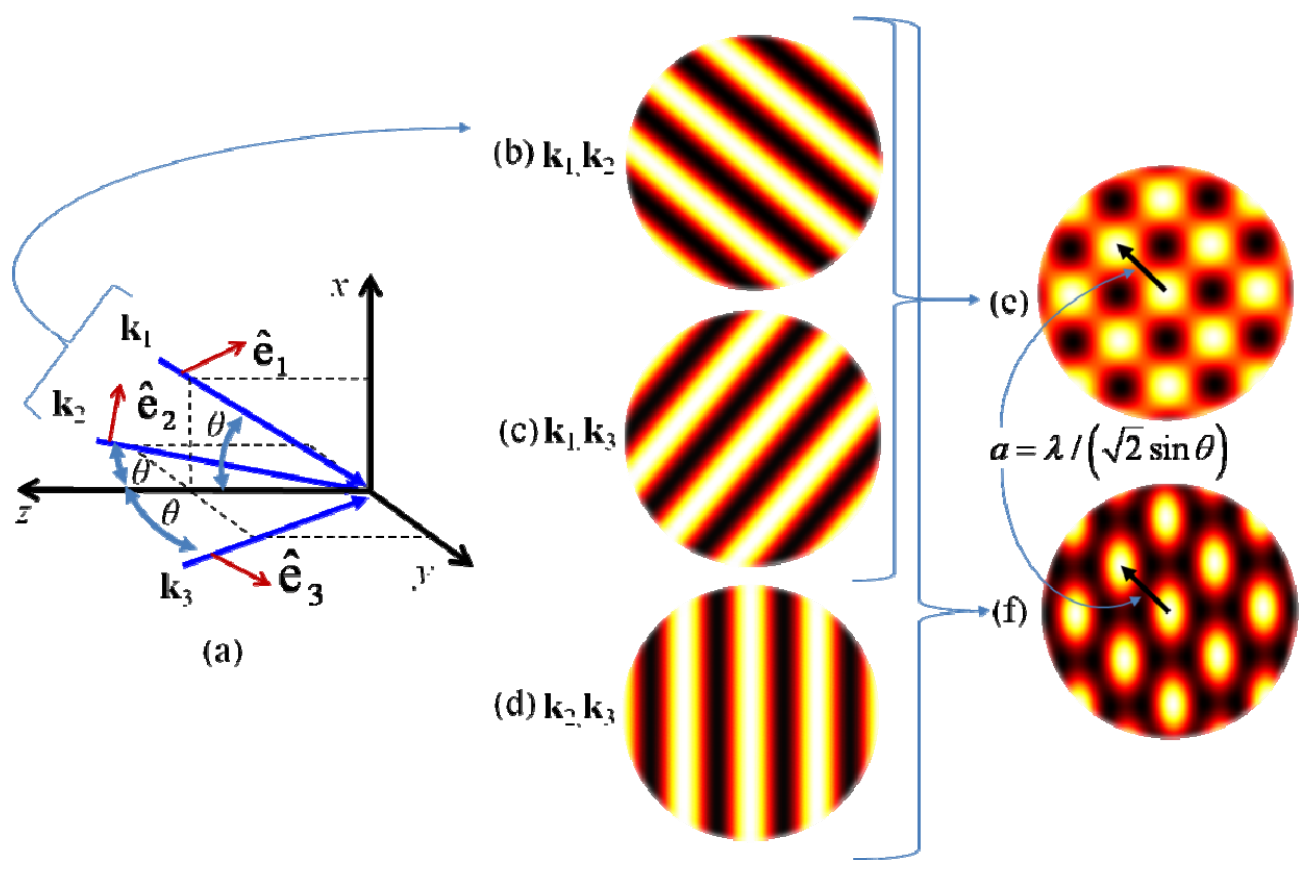

\subsection{Advances in Multi-Beam Interference Periodic Patterning}

By careful selection of the individual beam wavevectors, amplitudes, and polarizations, research has demonstrated the ability to create all 2D Bravais lattices [10], five of seventeen 2D plane group symmetries [11], and all 3D Bravais lattices via single- [12-15] and multiple-exposure [16,17] techniques. If elliptical polarization is allowed for the individual beams, 4 additional 2D plane group symmetries have been demonstrated for a three-beam configuration [11]. Figure 3(a) demonstrates a 2D hexagonal lattice with $p 6 m$ plane group symmetry formed by three-beam interference. Figure $3(\mathrm{~b})$ depicts a 3D face-centered-cubic lattice formed by four-beam interference. When the phase of the 
individual beams is considered, additional control over the interference pattern is possible. For patterns created by four or fewer linearly polarized beams, a change to the relative phase simply translates the interference pattern according to the translational symmetry. If more beams are added, the relative phase difference of the beam pairs will further define the unit cell structure leading to increasingly complex patterning possibilities and space group symmetries [15,18-20]. As an example, a 2D honey-comb structure formed by six-beam interference is depicted in Figure 3(c). Alternatively, a phase shift may be introduced by elliptical polarization in one or more of the interfering beams to produce compound lattices formed by as few as four beams [21-23]. Other quasi-periodic patterns demonstrated using MBI include: 2D structures with 5-, 10-, 12-, and 60-fold symmetries [24,25], connected $f c c$-like lattices [26], chiral-basis [23] and icosahedral [27] structures, as well as Archimedean-tiled and Penrose lattices [25].

Figure 3. MBI pattern examples. (a) $2 \mathrm{D}$ hexagonal lattice with $p 6 m$ plane group symmetry. (b) 3D body-centered-cubic lattice (reproduced with permission from [28]). (c) 2D honey-comb structure (reproduced with permission from [18], Copyright 2006, American Institute of Physics).

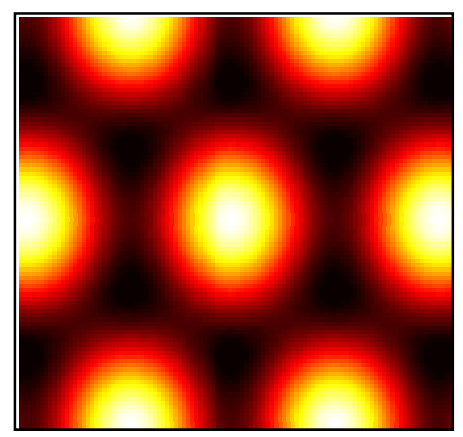

(a)

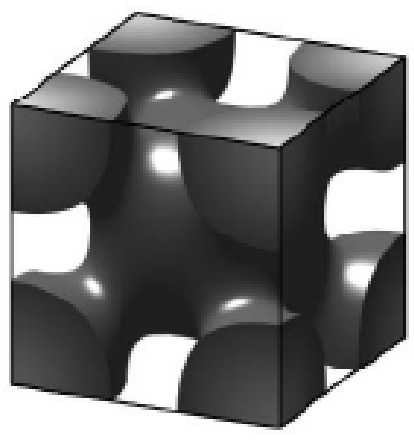

(b)

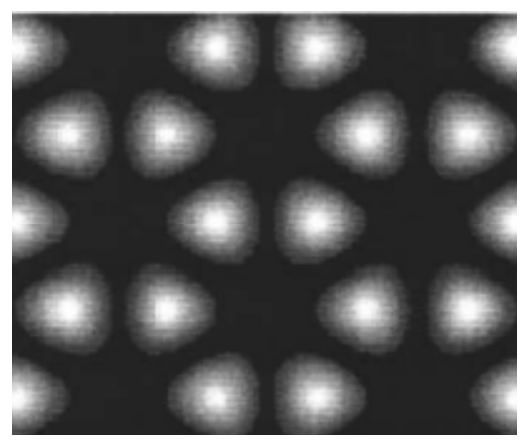

(c)

The translational periodicity together with the space group symmetry of the periodic patterns created by MBI determine the geometry of the individual lattice points, or motifs [29]. In some cases, the motif geometry plays a significant role in the performance characteristics of a device fabricated using MBI. For example, the lattice point geometry has been shown to affect the photonic-bandgap characteristics in photonic crystals [30-45], selective plasmonic excitation in plasmonic crystals [46], photonic crystal laser beam pattern [47] and polarization mode control [48], birefringence of photonic crystal fibers [49], cell behavior in tissue engineering [50], tuning of surface textures [51], magnetization switching in periodic magnetic arrays [52], and, negative refraction and superlensing in metamaterials [53,54]. Accordingly, several studies report analytical and computational methods to select individual beam parameters to change the motif orientation and shape $[34,42,55,56]$ from hemispherical to hemielliptical [57]; circular to triangular [58]; as well as, general structures including micro-cavities, micro-bumps, and rectangular bumps [51]. Most recently, research has focused on the exact analytical design and fabrication of motifs with 2D geometries that vary from the shape of an ellipse to that of a rhombus [59].

Given the numerous periodic patterning and motif geometry possibilities afforded by MBI, significant research has focused on maximizing the absolute contrast of the interference pattern by 
optimized selection of individual beam amplitudes, polarizations, and phases [60-67]. In this effort, the concept of uniform contrast was introduced, in which the relative amplitudes and individual-beam polarizations are optimized to ensure the highest contrast of the pattern as a whole for three- [68-70] and four-beam interference [71,72]. More recently, the conditions for primitive-lattice-vector-direction equal contrasts provided a complete treatment of contrast considerations in three- [73] and four-beam interference $[28,74]$. While these conditions assume individual control over beam amplitudes and polarizations, recent research suggests that sufficient contrast is still possible, even when beam parameters are perturbed [22] or requirements are simplified by design constraints $[59,75]$.

\subsection{Multi-Beam Interference Configurations}

Numerous optical configurations have been employed to generate the multiple beams required for MBI with varying levels of control over beam parameters, interferometric stability, and coherence requirements. These configurations may be broadly categorized as amplitude- or wavefront-splitting methodologies.

Amplitude-splitting configurations typically divide the single common source beam into two or more beams through the use of beam splitters or other diffractive elements. The beams are then directed to intersect at the plane of interference through the use of mirrors, lens, and/or prisms. An example two-beam configuration to record a 1D grating utilizing a single beam splitter and beam-directing mirrors is depicted in Figure 4(a). In any two-beam configuration, multiple exposures are required to generate more complex $2 \mathrm{D}$ and $3 \mathrm{D}$ patterns in a photo-sensitive material [76-79]. This is often accomplished by incorporating a rotating sample stage at the recording plane [80-83]. More advanced amplitude splitting systems, such as the configuration depicted in Figure 4(b), allow for the single-exposure of multiple beams [84,85]. Ideally suited for research and development, these configurations often afford the option of individual control over beam amplitude, polarization, and phase, along with a wide range of wavevector configurations and incidence angles [86,87]. For example, using an argon-Ion laser source of $363.8 \mathrm{~nm}$, the configuration in Figure 4(b) can produce five of the seventeen 2D plane group symmetries with a lattice constant ranging from approximately $3.5 \mu \mathrm{m}$ down to $300 \mathrm{~nm}$. One drawback of these beam-splitting-based configurations is the potential for interferometric instability. Any perturbations to the optical components, optical path lengths, or relative phases of the interfering beams may result in a translation of the pattern or change in the symmetry of the unit cell as discussed earlier.

To lessen these effects, other amplitude-splitting configurations have been developed that rely on gratings to diffract a single incident beam into two or more beams. These beams are, in turn, focused onto the plane of interference by either a lens system as depicted in Figure 4(c) $[72,88]$, beam-directing mirrors $[25,89]$, or cascaded diffractive elements $[90,91]$ such as the achromatic interference lithography configuration depicted in Figure 4(d) [92]. The symmetries formed by the zero- and first-order diffracted beams in Figure 4(c) make this configuration particularly useful in implementing the much-studied five-beam umbrella configuration consisting of a central beam (C-beam) surrounded by four symmetric ambient beams (A-beams) [67,93-96]. For an umbrella configuration, this scheme is particularly useful if the individual beams require specific conditioning, such as different polarizations (e.g., circularly polarized C-beam and linearly polarized A-beams) [97] or phase control [25]. 
Figure 4. Amplitude-splitting MBI configurations. (a) Two-beam configuration used to record a subwavelength polarization-dependent reflection grating (edited with permission from [98]). (b) Three-beam MBIL configuration for single-exposure 2D patterning with individual control of beam amplitudes and polarization (edited with permission from [99], Copyright 2011, American Institute of Physics). (c) Five-beam MBIL configuration for single-exposure patterning with a diffractive beam splitter (DBS), confocal lens system (edited with permission from [88], Copyright 2001, American Institute of Physics). (d) Cascaded phase gratings to implement achromatic interference lithography (edited with permission from [92], Copyright 1995, American Vacuum Society).

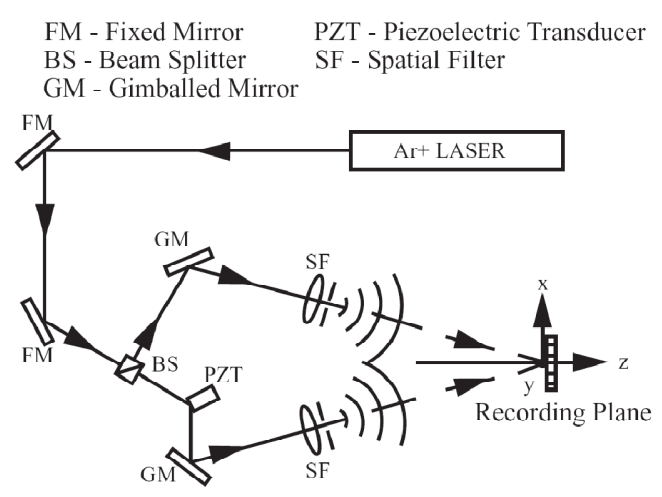

(a)

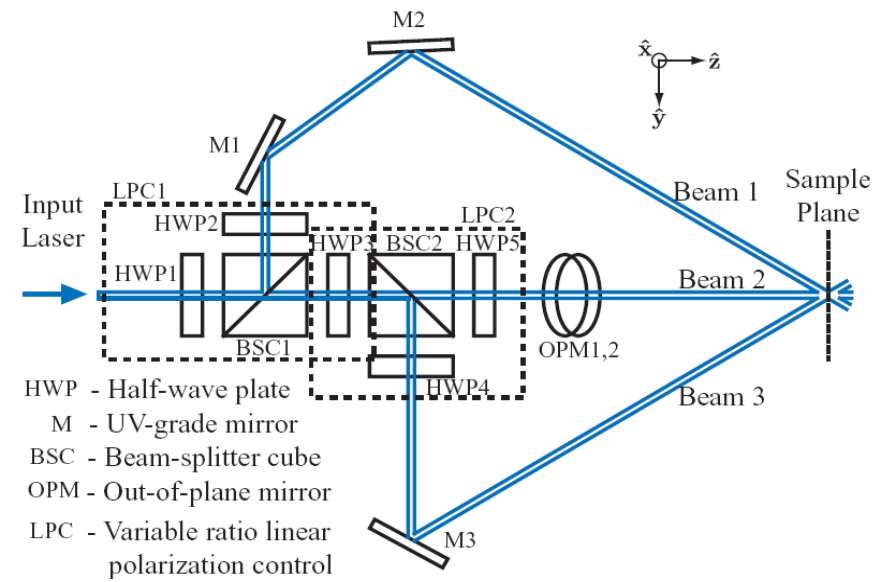

(b)

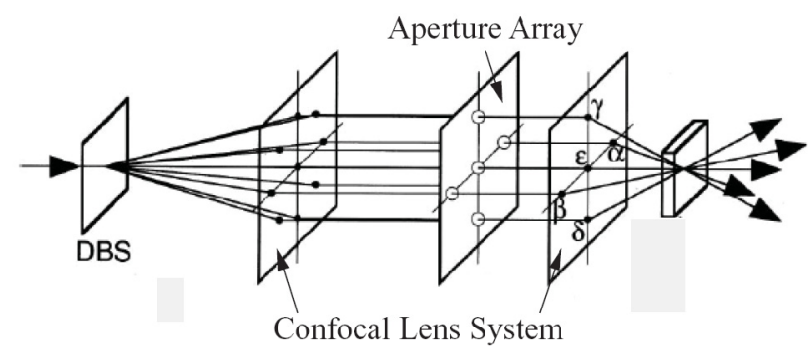

(c)

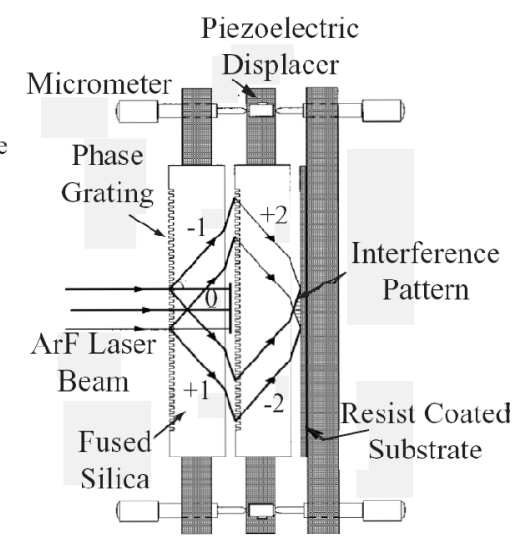

(d)

To simplify optical configurations and improve interferometric stability, wavefront-splitting schemes have been developed that divide portions of a single expanded source beam into the multiple beams required for MBI. The most common and widely used wavefront-splitting configuration incorporates a Lloyd's mirror to reflect a portion of an expanded source beam to intersect with the transmitted portion as depicted in Figure 5(a). As early as 1985, a Lloyd's mirror configuration was used as a simple way to fabricate linear grating couplers [100]. Again, multiple exposures are required with this configuration to produce 2D and 3D patterns [101], with one recent report of a Lloyd's mirror capable of producing three beams with 120 degree symmetry [102]. A Lloyd's mirror configuration may also be combined with immersion lithography, further reducing the fringe pattern periodicity to the sub-100 $\mathrm{nm}$ range [103]. 
Figure 5. Wavefront-splitting MBI configurations. (a) Lloyd's mirror configuration reflects a portion of the incident beam onto the transmitted beam at the sample. (b) A prism is used to divide and refract different portions of an incident collimated beam to produce four-beam umbrella interference (edited with permission from [136], Copyright 2005, American Institute of Physics). (c) A diffractive photo-mask diffracts portions of the incident expanded beam such that the first order diffracted beams intersect and interfere at the sample plane (edited with permission from [112], Copyright 2001, American Institute of Physics). (d) A 1D phase mask diffracts the incident beam into $+1,0$, and -1 diffracted orders to create a near-field interference pattern (edited with permission from [123]). (e) A double-iris amplitude mask defines two MBIL beams from a single collimated source for multiple two-beam interference patterning (edited with permission from [133]).

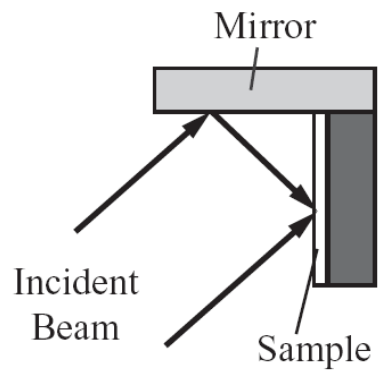

(a)

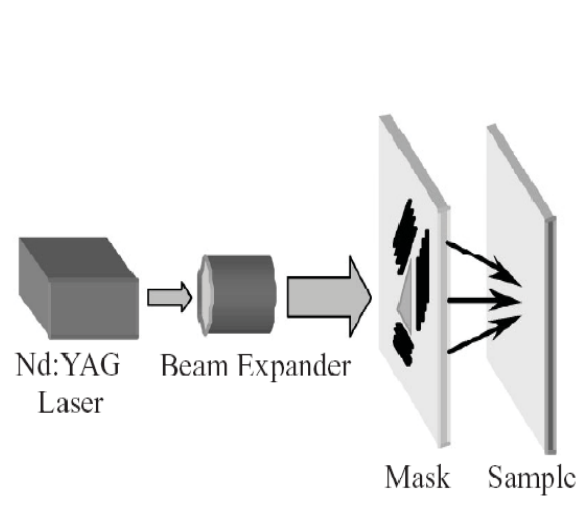

(c)

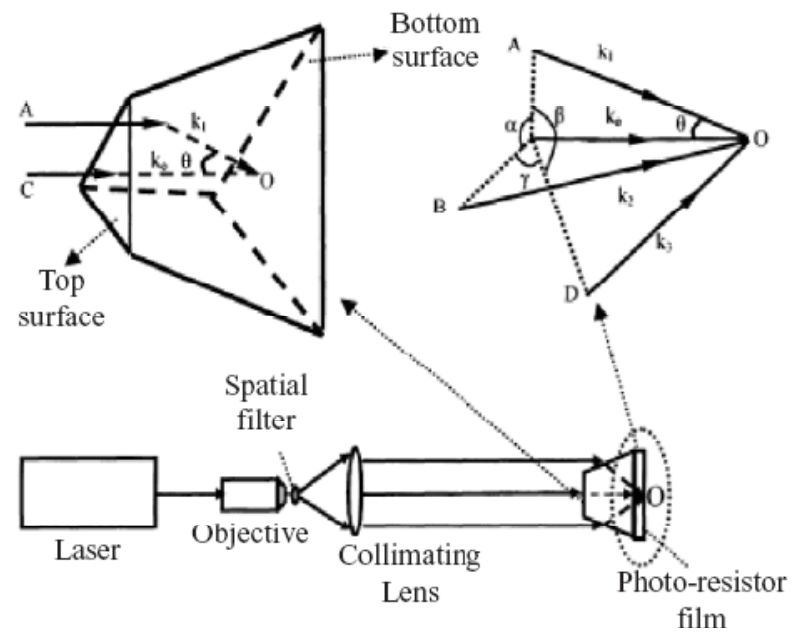

(b)

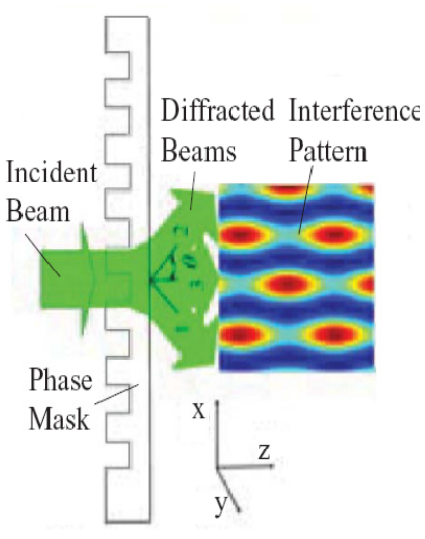

(d)

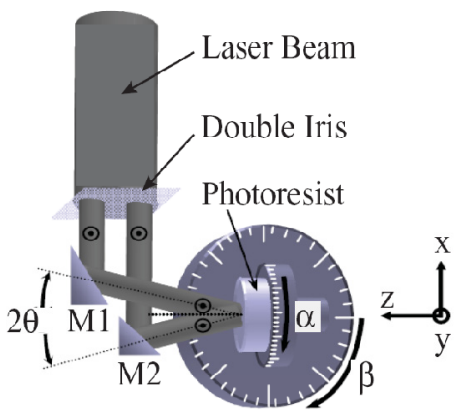

(e)

A second common wavefront-dividing configuration incorporates a specially designed prism to divide and refract the single expanded beam into multiple beams as depicted in Figure 5(b) [104-106]. This prism-based method is particularly useful in implementing the previously discussed umbrella configuration, with expanded options for four or more beams [107]. By selecting a prism with a refractive index matching that of the recording material, refraction-induced asymmetries may be mitigated at the at the prism-sample plane interface. In fact, an index-matching prism element may be 
also be used in amplitude-splitting configurations, incorporated at the sample plane, to mitigate these same asymmetries, including changes to the individual beam polarizations at the air-photoresist interface [25,108,109].

Diffractive gratings may also be used in a wavefront-dividing scheme in the form of a single diffractive photo-mask. In this configuration, two or more gratings are typically used to diffract a single expanded beam, such that the first order diffracted beams intersect and interfere at the sample plane as depicted in Figure 5(c) [110-115]. In one report, multiple gratings with precisely controlled periods were used to produce compound periodic patterns with a large depth of focus using an achromatic source [19]. If an umbrella configuration is required, the zero-order diffracted beam from a diffractive photo-mask may function as the C-beam [116].

A fourth option for wavefront splitting places a photo mask with a single diffractive optical element in close proximity to the sample plane to produce a near-field self-interference pattern of the zero-, positive-, and negative-diffracted orders as depicted in Figure 5(d) [117-121]. In this example, a 1D phase mask diffracts the incident beam into three interfering beams at the exit of the mask. Multiple exposures of a 1D phase mask may be used to produce complex 3D periodic patterns, such as a woodpile-type structure $[122,123]$. Alternatively, a multi-layer mask with two orthogonal diffractive gratings [124-126] or a single 2D diffractive optical element [127-130] may be used to produce multiple beams with a single exposure. As a result of the relatively short optical path lengths for the diffracted beams, this method is essentially phase-locked, representing the most interferometrically stable option for MBIL. However, with this advantage does come some disadvantages. As with the previous three wavefront-splitting configurations, control over individual beam amplitudes and polarizations is typically limited, wavevector configurations are generally fixed, and each are sensitive to transverse incoherence. An amplitude-splitting configuration with even-symmetry reflections or diffractions as depicted in Figure $4(\mathrm{a}, \mathrm{c}, \mathrm{d})$ may be used to resolve transverse coherence issues, assuming any beam offset is within the transverse coherence length and the spatial coherence meets depth of focus requirements [131,132].

Finally, a fifth wavefront-splitting option is depicted in Figure 5(e), employing a double-iris amplitude mask to define the individual beams, while providing the opportunity for individual beam conditioning and wavevector control [133-135].

\subsection{Multi-Beam Interference Lithography and Nano-Electronics}

As early as 1970, the fringe patterns formed by two-beam interference were recorded in photoresist, forming a simple, reproducible diffraction grating for use in thin-film waveguides [137]. Two decades later, multiple MBIL exposures were proposed to generate more complex 2D patterns in a photoresist [138]. Since then, a wide range of structures have been recorded via MBIL using near-infrared [129,139-142], visible light [18,31,32,62,88,143-153], ultraviolet (UV) [62,77,78,99,115,154-160], deep-UV [92,101,142,161-163], and extreme-UV sources [164-167].

Today, MBIL is considered a potentially key enabling technology for the ever-increasing demand for smaller feature sizes in optical lithography $[4,131,168]$. Modern integrated circuits have very regular layouts with an underlying grid pattern that defines the smallest feature size in the integrated circuit. MBIL can be used to define this underlying periodic or quasi-periodic grid-based structure. In 
this application, MBIL immediately has the advantages of (1) simple optics, (2) large working distances, (3) fast processing, (4) low cost, and (5) extendable to higher resolutions [4,25,169-171].

To create functional elements in the periodic pattern defined by MBIL, a second lower spatial-frequency trim exposure may be used to define the non-periodic features using a conventional technique such as projection lithography (PL), thereby easing the diffraction limitations of using PL alone [2,5,172]. As an example, Figure 6(a) depicts a hybrid optical maskless lithography technique demonstrating the use of two-beam interference to form vertical fringes, followed by a second traditional low-pitch PL trim exposure [163]. Similarly, Figure 6(b) demonstrates a multi-step procedure to create more complex composite patterns. Others have combined MBIL with various lithographic techniques including other mask-based techniques (e.g., proximity or contact lithography) $[159,173]$, electron-beam lithography [81,174,175], electron-beam-induced deposition [176], focused ion-beam lithography [155,177], direct laser writing [77,150,178,179], atomic force microscopy nano-indentation [180], and multi-photon polymerization [181-183]. For example, interference lithography has been combined with optical contact lithography to fabricate triple-gate metal-oxide-semiconductor field effect transistors [161]. In another report, interference lithography was used for metal thin-film patterning to fabricate the electrodes and metallization patterns required by modern microelectronic devices [139]. In fact, a recent economic assessment of various lithography choices indicates that a double-exposure technique, optimized by the use of MBIL, is the best choice for low to medium volume products [4]. Future research could make MBIL a preferred component of higher volume production as well.

Figure 6. Multi-beam interference lithography. (a) Scanning electron microscope image of the combined use of MBIL and projection lithography techniques. Here, $45 \mathrm{~nm}$ grid lines were produced via two-beam interference lithography. Next, the higher spatial frequency modulating pattern was recorded via projection lithography (Copyright 2004, Reprinted with permission of Cambridge University Press [2]). (b) A complex composite pattern is created by two MBIL exposures and two projection lithography trim exposures (edited with permission from [5]).

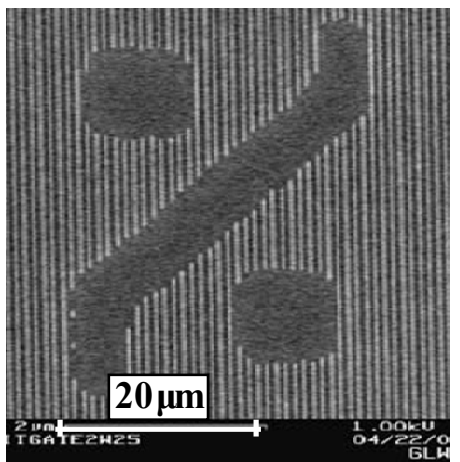

(a)

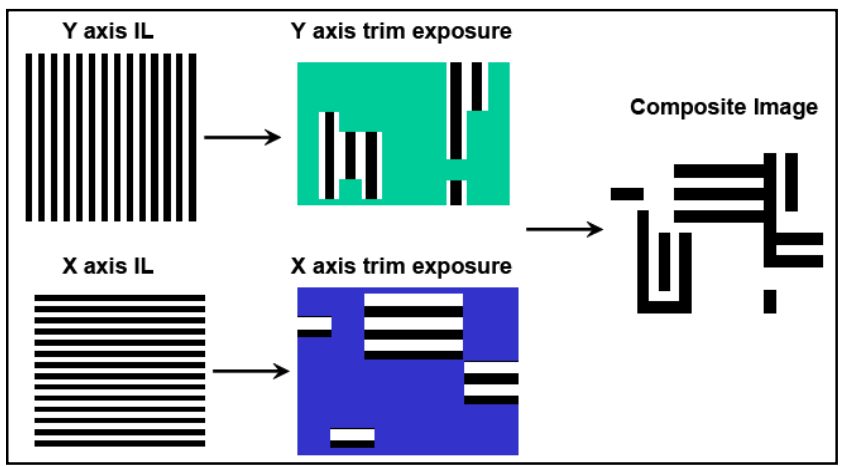

(b)

A wide array of photosensitive materials have been used to record the interference patterns formed by MBIL and include positive resists [115,157,184], negative resists [99,185], hybrid organic-inorganic materials [165,186,187], extreme-UV photoresists [188], silsesquioxane-based photoresists [129], 
holographic polymer-dispersed liquid crystals [189], amorphous-chalcogenide-semiconductor thin films [190], titanium-containing monomer films [191], red-sensitive photopolymers [145], polyimide foils [51], biocompatible polymers [50], oligomer films [153], and even silica [192] and chalcogenide [193] glasses. In the most general terms, given sufficient optical intensity, an interference pattern may be recorded in or on any material that responds to laser illumination at a given wavelength [160], to include direct writing on metallic surfaces via laser interference metallurgy [194] and direct laser interference patterning of $\pi$-conjugated polymers [195].

With the numerous patterning options, simple optical configurations, and opportunities in optical lithography, MBI has found applications across a wide range of technological areas beyond nano-electronics, including photonic crystals, metamaterials, subwavelength structures, optical trapping, and biomedical structures. Thus, a unified review of these broad applications follows.

\section{Photonic Crystals}

Perhaps the most documented application of MBI and MBIL has been in the formation of photonic crystal (PC) structures and is the subject of more than half of the references included in this review. PC technology has many important possible commercial applications [196,197] with the possibility of lossless control of light propagation at a size scale near the order of the wavelength of light. This technology has the potential to produce the first truly dense integrated photonic circuits and systems (DIPCS). Individual components that are being developed include resonators, antennas, sensors, multiplexers, filters, couplers, and switches. The integration of these components would produce DIPCS to perform functions such as image acquisition, target recognition, image processing, optical interconnections, analog to digital conversion, and sensing. Further, the resulting DIPCS would be very compact in size and highly field portable. Applications using light at telecommunications wavelengths require structures to be fabricated with nano-sized dimensions. Despite the advantages and benefits of using such a technology in commercial devices, a major problem exists: the practical commercial development of PC structures has been very slow.

To date, research has not fully developed a methodology for the large-scale and cost-effective integration of these impressive PC-based devices into manufacturable DIPCS. No rapid and inexpensive systematic fabrication procedure has been developed for the reliable and reproducible fabrication of nano-sized PC structures. MBIL has been proposed as one method to overcome this deficiency. In the late 1990s, interference lithography was used to produce 2D [158] PC lattices exhibiting a photonic bandgap and later 3D face-centered cubic [157] PC structures as depicted in Figure 7(a). Since then, MBIL has been extensively studied, optimized, and used to fabricate PCs [109,184,198-203] with ongoing efforts to develop PC-based devices through a combination of lithography techniques such as the PC waveguide depicted in Figure 7(b). In fact, the design rules for MBIL-defined PC waveguide devices have been theoretically defined, when interference lithography is combined with direct laser writing [204]. Recently, nanoscale photonic-crystal-band-edge lasers were fabricated with the use of interference lithography, paving the way for the integration of electrically-driven PC lasers [205] as depicted in Figure 7(c). Other proposed PC-based devices relying on MBIL include optical add-drop multiplexers [206], organic light emitting diodes (LEDs) [207], coupled PC resonator arrays [208], and PC distributed feedback quantum cascade lasers [209]. 
Figure 7. Photonic crystals (PC). (a) 3D face-centered-cubic PC structure created by four-beam MBIL. Inset $\mathbf{A}$ shows a scanning electron microscope image of the bottom surface of the PC, while B depicts the reconstructed 3D surface (Reprinted by permission from Macmillian Publishers Ltd: [157], Copyright 2000). (b) PC waveguide created by electron-beam lithography in a 2D hexagonal lattice formed by UV MBIL (edited with permission from [174]). (c) Schematic view of an air-bridge type PC band edge laser with scanning electron microscope images of the square lattice PC structure fabricated using MBIL (edited with permission from [205], Copyright 2005, American Institute of Physics).

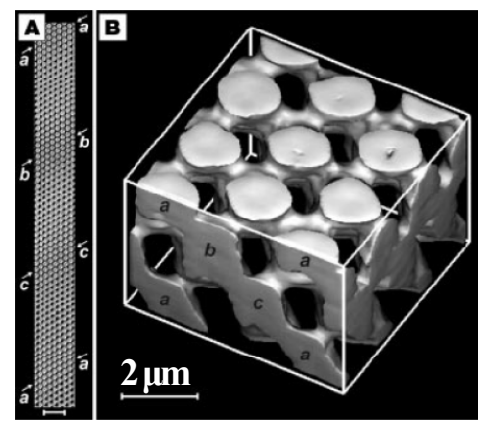

(a)

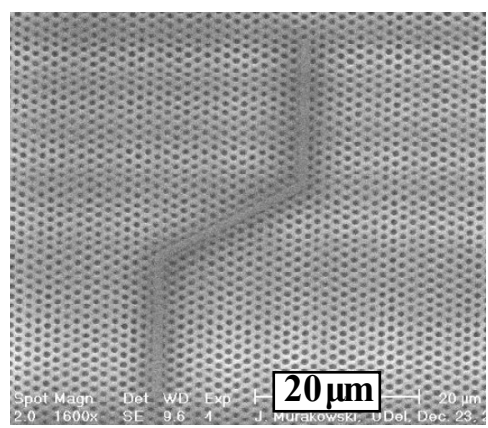

(b)

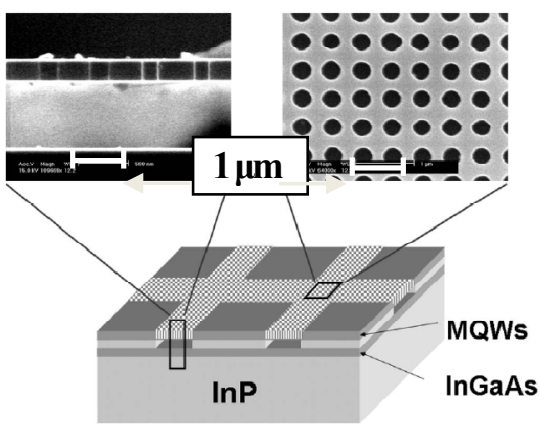

(c)

\section{Metamaterials}

Like photonic crystals, metamaterial technology offers the control of light propagation, only now at a size scale much smaller than the wavelength of light. Accordingly, metamaterial-based devices have many important possible commercial applications [210-213]. Ultra-compact objective lenses, frequency-doubling devices, parametric amplifiers, electromagnetic cloaking, and parametric oscillators all become possible with metamaterials. The integration of these components would produce DIPCS that are very compact in size and highly field-portable.

In natural materials (as contrasted to metamaterials), atoms are arranged in a regular pattern with periods of approximately half of a nanometer. In these natural materials, the electric field component of the incident light excites the electric dipoles of the material. These dipoles re-radiate with some phase retardation. Thus, the electric relative permittivity $\varepsilon_{\mathrm{r}}$ is not equal to unity. In contrast, the magnetic dipoles do not appreciably interact with the magnetic field component of the incident light. Thus the relative magnetic permeability $\mu_{\mathrm{r}}$ is unity. Metamaterials overcome this lack of magnetic dipole through subwavelength-sized artificial magnetic dipoles (e.g., split-ring resonator structures). The index of refraction, in general, is $n= \pm\left(\varepsilon_{\mathrm{r}} \mu_{\mathrm{r}}\right)^{1 / 2}$. For the case when both $\varepsilon_{\mathrm{r}}$ and $\mu_{\mathrm{r}}$ are less than zero, the refractive index is negative. That is, $\mathrm{n}=-\left(\varepsilon_{\mathrm{r}} \mu_{\mathrm{r}}\right)^{1 / 2}$. The index is not $\mathrm{n}=+\left(\varepsilon_{\mathrm{r}} \mu_{\mathrm{r}}\right)^{1 / 2}$. This result was first reported and discussed by Veselago [214].

The subwavelength-sized magnetic dipoles needed to make a metamaterial, can be produced by making microscopic split-ring resonators (SRR's). These SRR devices have also been called "slottedtube resonators" or "loop-gap resonators" in the literature. If SRR devices are produced in subwavelength arrays, they behave as "magnetic atoms" and thus metamaterials become possible. Metamaterials have been successfully developed at microwave frequencies [215-217]. However, the production of metamaterials at optical frequencies has been much more challenging [218,219]. Further, 
current technologies lack the ability to produce these precise nanostructure devices rapidly and at low cost. Again, MBIL provides a potential solution to this critical shortcoming [220]. To produce a large area split-ring array template for magnetic metamaterials, MBIL has been proposed as a mechanism to form single-slit and double-slit split-ring arrays in 2D triangular and square lattice arrays [221]. Others have used MBIL to demonstrate high-throughput experimental fabrication of metal-dielectric-metal "magnetic atoms" [222,223] as depicted in Figure 8(a), as well as cylindrical nanoshell, U-shaped, and double-split ring resonator array metamaterials [224] as depicted in Figure 8(b). In some cases, MBIL may be used in conjunction with other methods to facilitate the economical fabrication of metamaterials. For example, interference lithography can be used to form a 1D array of trenches to serve as a template for the self-directed assembly of a silver nanocluster metamaterial [225].

Figure 8. Metamaterial examples. (a) Scanning electron microscope view of a "magnetic atom" defined by a three-beam-generating MBIL prism, with an oblique-incidence view of layered $\mathrm{Au}$ (golden), $\mathrm{MgF}_{2}$ (blue), and $\mathrm{Au}$ (edited with permission from [222]). (b) Scanning electron microscope view of double-split ring resonator array metamaterial fabricated via phase-modulated six-beam MBIL (edited with permission from [224]).

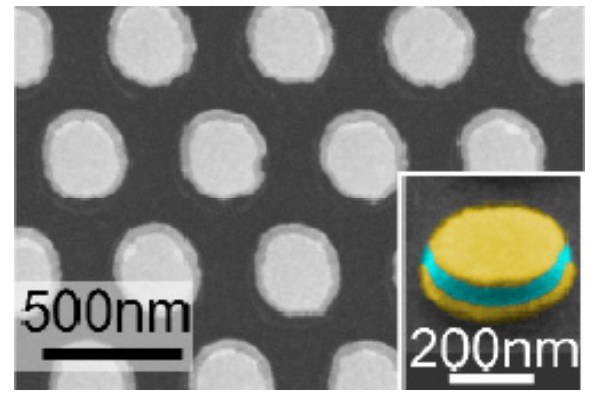

(a)

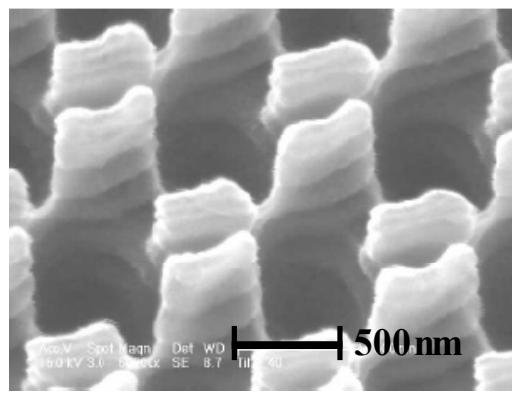

(b)

\section{Subwavelength Structures}

Research into micro- and nano-scale subwavelength structures has grown exponentially in the last decade. By utilizing the subwavelength periodic patterns produced by MBIL, numerous important structures can be realized for an increasing number of subwavelength structure applications, including synthesized-index elements, form-birefringent polarization elements, guided-mode resonant elements [226], field-emission devices, plasmonic structures, surface texturing, magnetic nanostructures, and numerous other nanotechnology efforts.

\subsection{Synthesized-Index Elements}

Synthesized-index elements include 1D and 2D periodic surface structures for use as antireflection surfaces. Simple 1D binary surface-relief gratings on dielectrics can exhibit zero-reflectivity at optical wavelengths [227,228]. For example, interference lithography has been used to record subwavelength gratings on the surface of diffractive beam splitters, reducing surface reflections, thereby improving efficiency [229]. Similarly, MBIL was used to fabricate subwavelength antireflective coatings to improve the performance of optoelectronic LEDs [230]. More recently, subwavelength antireflection patterns were recorded in a $\mathrm{Si}$ substrate using interference lithography, providing broadband 
(250-1,200 nm), wide angle $\left(0-45^{\circ}\right)$ operation [231] as depicted in Figure 9(a). Such antireflection surfaces have been extended to lossy, even metallic, materials [232,233]. 2D crossed gratings have also been implemented to provide polarization-independent antireflection behavior, mimicking the effect of a "moth's eye" [149,234,235]. Periodic and quasi-period surfaces have been widely applied to increase the efficiency of solar cells [236,237]. To this end, interference lithography was recently used to fabricate antireflective gratings on thin-film solar cells [238]. Other example applications include a $100 \mathrm{~nm}$ period grating in an amorphous silicon thin film used to stabilize the linear polarization of vertical-cavity surface-emitting laser operating at $850 \mathrm{~nm}$ [239], and a critical angle transmission grating facilitated by the use of scanning-beam interference lithography [240].

Figure 9. Subwavelength optical structures. (a) Synthesized-index anti-reflective Si post array defined by multi-exposure MBIL (edited from [231], Copyright 2010, with permission from Elsevier). (b) Form-birefringent polarization grating retarder fabricated via two-beam interference (edited with permission from [98]).

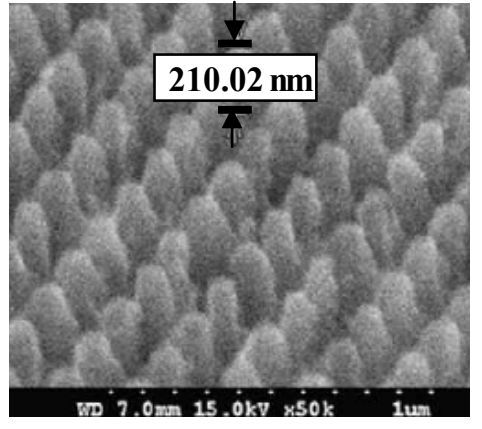

(a)

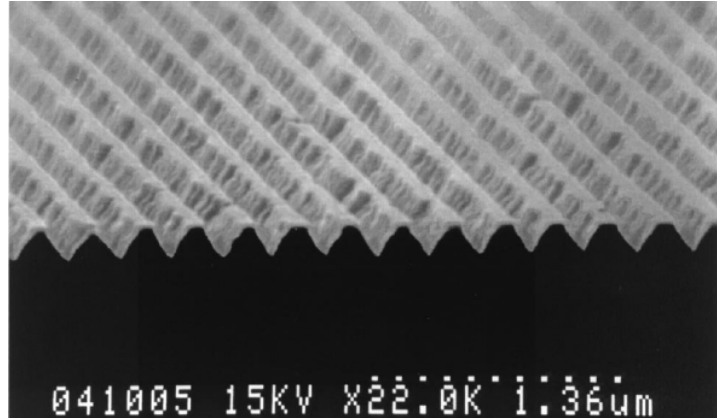

(b)

\subsection{Form-Birefringent Polarization Elements}

Form-birefringent polarization elements include retardation plates, polarizers, and beam-splitters. Retarders can behave as quarter-waveplates, half-waveplates, full-waveplates, etc. and thus convert linearly polarized light to circular polarization, rotate linear polarization, provide narrowband filtering, etc. Subwavelength grating retarders have been made using MBIL for use in the visible and in infrared regions of the spectrum [241] as depicted in Figure 9(b). Similarly, subwavelength-grating polarizers have also been reported [98]. Two-beam interference lithography has been demonstrated as a highly cost effective fabrication process to generate these subwavelength structures [228].

\subsection{Guided-Mode Resonant Elements}

Guided-mode resonant gratings are widely utilized for their very narrowband filter characteristics [242-244]. Such subwavelength-grating filters represent important devices in dense-wavelength-division multiplexed communications and in chemical, biological, toxicological, and environmental sensing applications. Recently, broadband versions of these resonant gratings have also been demonstrated [245]. 


\subsection{Field Emission Devices}

The process of field emission ejects electrons from a metal surface or a doped semiconductor material under the influence of an applied electric field. Traditionally, field emission is generated from micro-structured tips used as the cathodes for electron extraction. Field emission devices have found applications in field emission displays, vacuum microelectronics, satellite subsystems, mass spectrometers, and even electrodynamic space tethers [246,247]. As early as 1995, interference lithography was used to manufacture high density micro-field emitter arrays with submicron emitter spacing to improve field emission display performance and reduce manufacturing costs [156,248]. Recent efforts have focused on the use of interference lithography, combined with catalytic etching, to produce the nanocones used in field emission as depicted in Figure 10(a), potentially eliminating the need for other more complicated lithography techniques [249]. Others have employed MBIL to aid in the production of carbon nanotubes as depicted in Figure 10(b) that could prove useful in field emission display applications [250].

Figure 10. Field emission structures. (a) Scanning electron microscope view of silicon-etched nanowires defined by two-beam, multiple-exposure MBIL (edited with permission from [249], IOP Publishing Ltd). (b) Tilted scanning electron microscope view of vertically-aligned carbon nanofibers, where a multi-exposure Lloyd's mirror configuration provides the Ni catalyst patterning for nanofiber growth (edited from [250], copyright 2010, with permission from Elsevier).

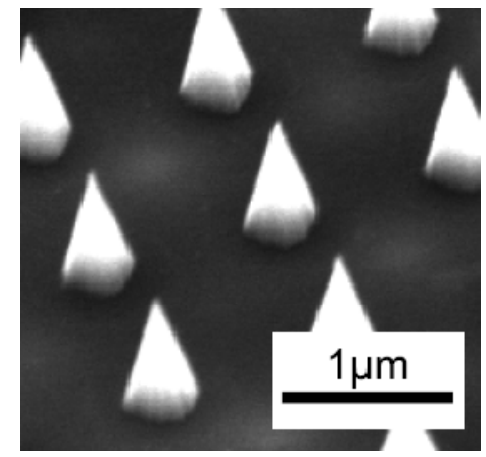

(a)

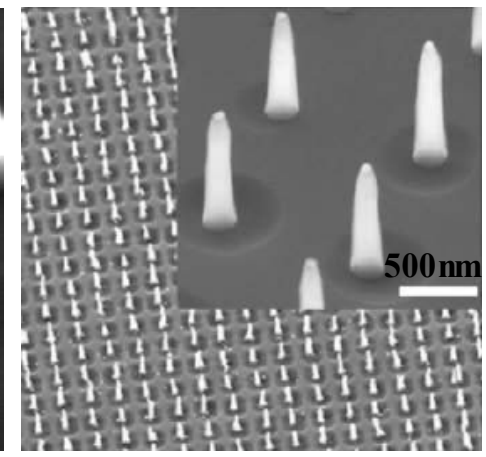

(b)

\subsection{Plasmonic Structures}

Surface plasmon resonances occur for a range of frequencies when an electromagnetic field excites oscillations of free electrons on the surface of metallic nanostructures [251]. Near-field coupling effects on the plasmon resonance of these metal nanostructures, called localized surface plasmons, can be used in biosensing applications, surface enhanced Raman spectroscopy, and novel photonic devices [82,252,253]. The mechanism of plasmonic resonance is directly related to the refractive index variation in the metallic film nanostructures, commonly fabricated using self-assembly methods. As an alternative to self-assembly methods, MBIL has been used as a mechanism to produce plasmonic metallic nanostructures including silver dot arrays [251,254], gold particle arrays [82,252], and bimetallic dot structures [46,253] depicted in Figure 11(a-c) respectively. 
Figure 11. Plasmonic structures. (a) Scanning electron microscope image of MBIL-defined hexagonal lattice of elliptical holes in a $100 \mathrm{~nm}$ thick silver film (edited with permission from [254]). (b) 3D view of gold nano-particle array with a lattice constant of $400 \mathrm{~nm}$, defined by two-beam, multi-exposure MBIL (edited from [82], Copyright 2009, with permission from Elsevier). (c) Scanning electron microscope image at a 50 degree tilt of bimetallic $(\mathrm{Ag} / \mathrm{Au})$ dots on a quartz substrate fabricated using a Lloyd's mirror configuration (edited with permission from [253]).

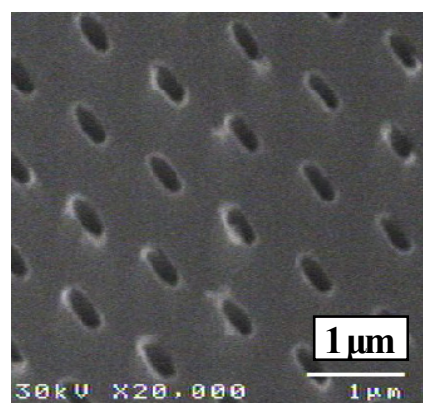

(a)

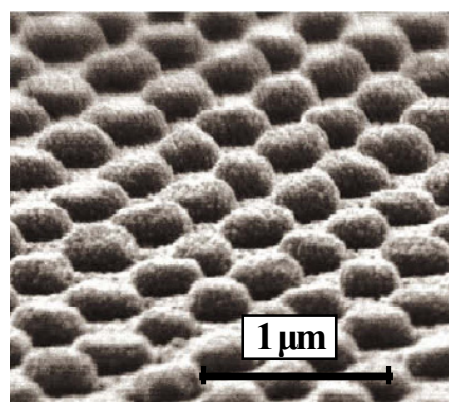

(b)

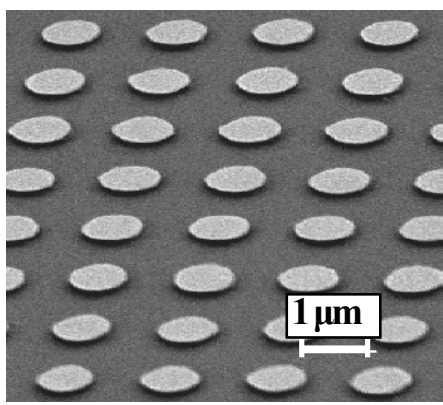

(c)

\subsection{Surface Texturing}

Surface patterning at the micro- and nano-scale has been the subject of much study, with applications across numerous areas, including microelectromechanical systems, automotives, aviation, defense, and biological areas [194,255]. Laser surface texturing using MBI is of particular interest to tribology technologies including textured surfaces for mechanical seals, piston rings, and thrust bearings. In one study, laser interference metallurgy was employed to improve lubrication film lifetimes and the tribological behavior of various surface textured materials as depicted in Figure 12(a) [194]. Others have studied the wetting properties and superhydrophobicity of surfaces, patterned by laser interference, for such applications as corrosion inhibition, microfluidic technology, self-cleaning window glass, and evaporation-driven nanopatterning [151,256]. Similarly, interference patterning has been used to create biomemetic structures as depicted in Figure 12(b), mimicking natural sub-micron patterns [257] such as bone material and shells exhibiting advantageous mechanical stability and dynamic surface properties [255].

\subsection{Magnetic Nanostructures}

Magnetic nanostructures, often called "nanomagnets," have been proposed as an alternative technology for information storage [258,259]. In this application, discrete nanomagnets with uniaxial magnetic anisotropy store binary information when magnetized in one of two possible antiparallel directions. To generate these magnetic nanostructures, interference lithography has been used to generate a nanoscale array of dots that are, in turn, transferred to a magnetic film using ion beam etching as depicted in Figure 13(a) [258,259]. Others have employed interference lithography in the fabrication of cobalt magnetic arrays for use in magnetic random access memory devices as depicted in Figure 13(b) [260]. Most recently, MBIL was used to create magnetic patterns in Co/Pd multilayer systems with favorable magnetic properties as depicted in Figure 13(c) [261]. 
Figure 12. Surface texturing. (a) Scanning electron microscope view of laser interference metallurgy defined dot-like structures, recorded directly on a metallic surface with low laser fluence to change the tribological behavior of the surface (edited from [194]. Copyright Wiley-VCH Verlag GmbH \& Co. KGaA. Reproduced with permission). (b) MBIL-defined nanopilar arrays (inset B) are patterned in a hierarchical structure (inset A and inset B), mimicking the surface of a lotus leaf, for enhanced hydropholic phenomenon (edited from [257], Copyright 2010, with permission from Elsevier).

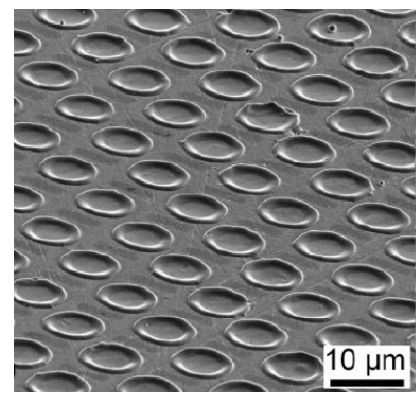

(a)

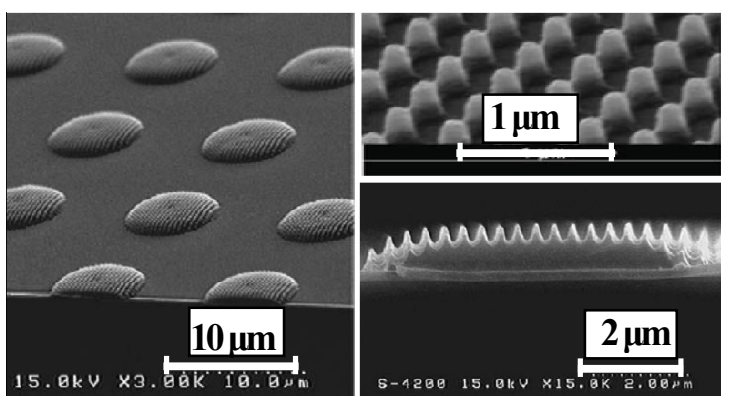

(b)

Figure 13. Magnetic nanostructures. (a) Scanning electron microscope profile image of a nanomagnet array with a periodicity of $400 \mathrm{~nm}$, defined by multiple exposures using a Lloyd's mirror configuration (edited from [259], Copyright 2005, with permission from Elsevier). (b) 1D cobalt array fabricated using two-beam interference (edited from [260], Copyright 2007, with permission from Elsevier). (c) atomic force microscopy topography image of a $\mathrm{Co} / \mathrm{Pd}$ multilayer system structured with three-beam MBIL (edited with permission from [261], Copyright 2009, American Institute of Physics).

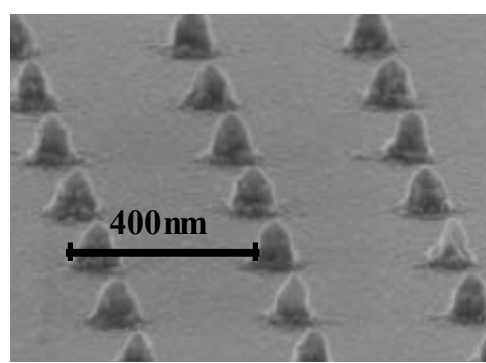

(a)

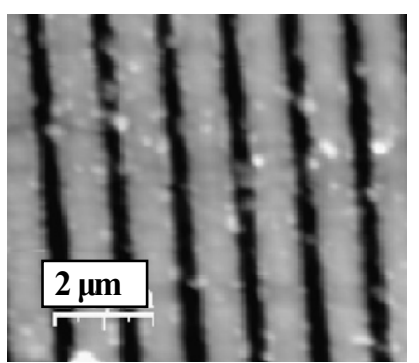

(b)

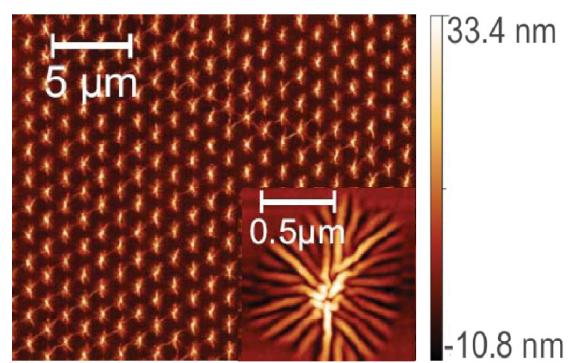

(c)

\section{Optical Trapping}

The periodic pattern produced by MBI need not always be recorded in a photosensitive material. For example, the light intensity distribution produced by MBI may be used directly as optical traps. Traditionally, optical trapping relies on the increased electrical field associated with the localized optical intensity of a focused beam. A dielectric particle is, in turn, guided by the increased electric field force to the point of the highest light intensity. By this mechanism, optical traps have been used to manipulate a range of particles including polymer spheres, metallic particles, and biological specimens [262]. Recently, techniques to manipulate suspended micro- and nano-scale particles have been realized through the use of holographic optical tweezers, trapping multiple objects simultaneously [263]. In this application, the focused beams are replaced by computer-defined beam 
arrays generated by a spatial light modulator. Using this method, sophisticated algorithms have been developed to provide updates to the computer generated hologram to control dynamically the orientation of the multiple trapped particles. Recently, this techniques was used to control and study rod-shaped bacteria [264] and zeolite L crystals [265].

As an alternative to computer generated holographic optical tweezers, MBI may provide similar optical arrays along with dynamic control of light intensity and orientations in two- and three dimensions, easing the requirement for complicated computer control algorithms. MBI optical trapping was first realized in the 1990's with a two-beam interferometric trap to hold polystyrene beads of a few microns in diameter as depicted in Figure 14(a) [266]. Later, 2D and 3D interference patterns were used to trap, sort, and manipulate trapped particles on a small scale using focused interfering beams [267-271] as depicted in Figure 14(c). Recently, large-area 3D arrays of optical traps have been demonstrated through the use of MBI, without the need for iterative computational algorithms, as depicted in Figure 14(b) [272].

Figure 14. Optical trapping. (a) Two-beam interferometric optical trap (edited from [266], Copyright 1997, with permission from Elsevier). (b) Image of a 19-sphere array of $2 \mu \mathrm{m}$ diameter silica spheres trapped in a hexagonal interference pattern formed by three-beam interference (edited with permission from [272]). (c) Experimental configuration for optical sorting in three-beam interference. The interference pattern is formed at the top of the glass curvette. Roman numerals indicate four different types of object sorting (edited with permission from [273], Copyright 2008, American Institute of Physics).

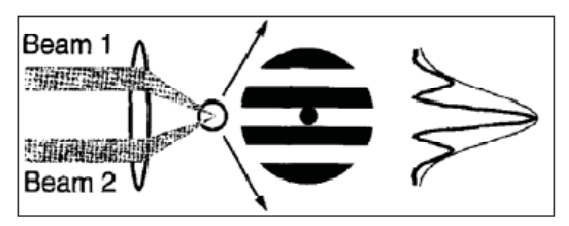

(a)

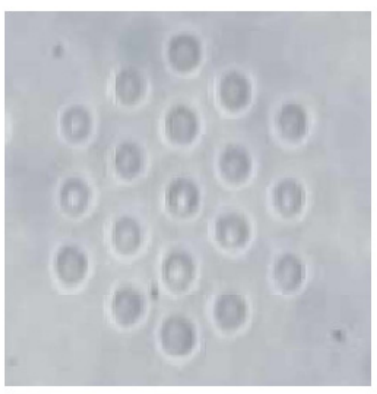

(b)

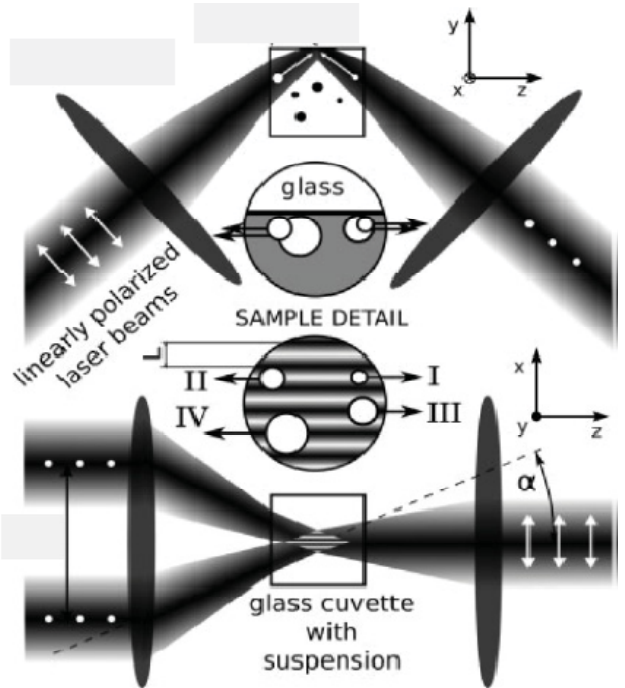

(c)

\section{Biomedical Structures}

Biomedical structures that are periodic or quasi-periodic in one-, two-, and three-dimensions are critically important in a wide variety of areas. Some representative example applications are described here to enable gauging the impact that they have in biomedicine. In regenerating nerves, arrays of microchannels are needed to guide nerve growth [274]. In facilitating bone regrowth, periodic meshes are needed to retain and sequester bone morphogenetic protein. This process reduces protein dose by 
localizing the morphogenetic stimulus [275]. In the forming, maintaining, and repairing of tissue, engineered surfaces are needed that present controlled densities of peptides to direct the assembly of extracellular matrices [276]. In measuring the strength of cell adhesion to the extracellular matrix, meshes are needed to control the size and position of cells to be able to determine the individual contributions of the various structures present [277]. In identifying genetic biomarkers for human disease, high density microarrays are needed for the detection of dozens of polymorphisms in a single analysis. For example, 11 micron square positions are used so that highly redundant oligonucleotide probes can ensure robustness [278]. In studying the functions of a cell (gene expression, adhesion, migration, proliferation, and differentiation), micropatterning of the cells is needed since the cell functions are affected by the microscale and nanoscale environment [279]. In enhancing bone formation in vivo, it is necessary to microstructure the titanium implant surfaces. For example, $100 \mu \mathrm{m}$ cavities are found to produce osteoblast attachment and growth [280]. In the controlled delivery of insulin, a permeable membrane mesh is needed that allows insulin and nutrients to pass through while blocking larger immune cells, T-cells, and antigen-presenting cells.

Figure 15. Biomedical applications. (a) Scanning electron microscope image of micro-structured particles formed using a combination of phase-mask MBIL and stop-flow lithography (Edited from [153]. Copyright Wiley-VCH Verlag GmbH \& Co. KGaA. Reproduced with permission). The inset shows the square-grid structure on the surface of the particles. (b) Scanning electron microscope image of square lattice of submicron domains indicate selective absorption of protein fibronectin that is confined to the MBIL-defined $100 \mathrm{~nm}$ domains indicated by a gold-enhanced solution (Edited from [282]. Copyright 2010 E. L. Hedberg-Dirk and U. A. Martinez). (c) Scanning electron microscope image of 3D hydrogel structure with interconnected nanopores fabricated using an MBIL phase mask and cross-sectional interconnects (inset) (Adapted with permission from [50]. Copyright 2008 American Chemical Society).

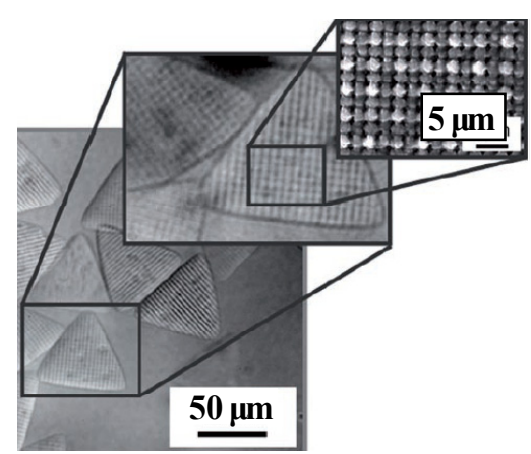

(a)

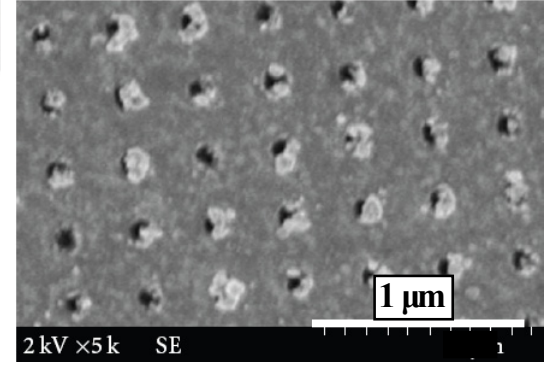

(b)

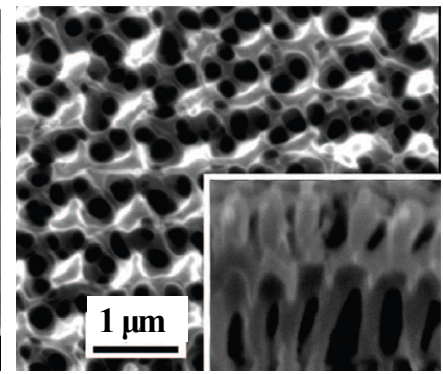

(c)

Common to all of these biomedical applications is the requirement for appropriate periodic or quasi-periodic structures that can be made by MBIL. This fact has led several to turn to MBIL for numerous biomedical applications in recent years. For example, micro-structured particles have been produced using a combination of MBIL and stop-flow lithography as depicted in Figure 15(a) with several potential applications including cell growth, tissue engineering, and biosensors [153]. To immobilize proteins in a square array of anchor sites, nano-scale lattices were formed by 
interference lithography. This application will assist in the analysis of biomaterials and the study of interactions of living cells with biomolecules [281,282]. As an example, Figure 15(b) depicts a scanning electron microscope image of square lattice of submicron domains to provide selective absorption of protein fibronectin, leading to advanced studies in the influence of protein organization in adhesion complex formation and function. In the development of neural prosthetic devices, phase mask interference lithography was used to fabricate 3D hydrogels with controlled geometries and sub-micron feature sizes as depicted in Figure 15(c). This application could lead to advances in drug delivery, tissue engineering and improved material-device interactions [50]. In the field of antibacterial research, MBIL was used to create a 2D pore-array to immobilize silver nanoparticles, known for their antibacterial properties. In a study of this application, the fabricated antibacterial nanocomposition proved effective against the E. coli bacteria [283]. Based on these recent examples, MBIL could become an important tool in the development of the next generation of biomedical structures.

\section{Conclusions}

MBI and MBIL have the potential to impact significantly a wide variety of applications. Numerous periodic and quasi-periodic patterns with specific space group symmetries and motif geometries are now possible by careful selection of individual beam amplitudes, polarizations, wavevector configurations, and phases. These same parameters also enable the optimization of the contrast of the resulting pattern, providing lithographically useful patterning possibilities. As such, several optical configurations and lithographic techniques have been developed to incorporate MBIL, providing the potential for low-cost, wafer-scale, simple, and fast lithographic fabrication. Of obvious interest to the nano-electronics industry, MBI and MBIL have found additional applications in the areas of photonic crystals, metamaterials, subwavelength structures, optical trapping, and biomedical structures

The potential importance of MBI continues to grow with the ever-increasing interest in nano-technologies. More research and development are needed to bring MBI into mainstream applications, including combining MBIL with other conventional processes to produce functional integrated devices. In doing so, MBIL may provide the technology necessary to continue to meet the predictions of Moore's law in the IC industry, while finding additional applications in an increasing list of micro- and nano-technology fields.

\section{Acknowledgement}

This research was supported in part by Grant No. ECCS 0925119 from the National Science Foundation.

\section{References}

1. Cressler, J.D. Silicon Earth: Introduction to the Microelectronics and Nanotechnology Revolution; Cambridge University Press: New York, NY, USA, 2009.

2. Rothschild, M. Nanopatterning with UV optical lithography. Mater. Res. Soc. Bull. 2005, 30, 942-946. 
3. Jang, J.H.; Ullal, C.K.; Maldovan, M.; Gorishnyy, T.; Kooi, S.; Koh, C.Y.; Thomas, E.L. 3D micro- and nanostructures via interference lithography. Adv. Funct. Mater. 2007, 17, 3027-3041.

4. Jhaveri, T.; Strojwas, A.; Pileggi, L.; Rovner, V. Economic assessment of lithography strategies for the $22 \mathrm{~nm}$ technology node. Proc. SPIE 2009, 7488, 74882Y.

5. Smith, B.W. Alternative optical technologies: More than curiosities? Proc. SPIE 2009, 7274, 727402.

6. Solak, H.H. Nanolithography with coherent extreme ultraviolet light. J. Phys. D Appl. Phys. 2006, 39, R171-R188.

7. Moon, J.H.; Ford, J.; Yang, S. Fabricating three-dimensional polymeric photonic structures by multi-beam interference lithography. Polym. Adv. Technol. 2006, 17, 83-93.

8. Xia, D.; Ku, Z.; Lee, S.C.; Brueck, S.R.J. Nanostructures and functional materials fabricated by Interferometric lithography. Adv. Mater. 2011, 23, 147-179.

9. Saleh, B.E.A.; Teich, M.C. Fundamentals of Photonics; John Wiley \& Sons, INC.: New York, NY, USA, 1991.

10. Cai, L.Z.; Yang, X.L.; Wang, Y.R. Formation of a microfiber bundle by interference of three noncoplanar beams. Opt. Lett. 2001, 26, 1858-1860.

11. Mao, W.D.; Zhong, Y.C.; Dong, J.W.; Wang, H.Z. Crystallography of two-dimensional photonic lattices formed by holography of three noncoplanar beams. J. Opt. Soc. Am. B 2005, 22, 1085-1091.

12. Cai, L.Z.; Yang, X.L.; Wang, Y.R. All fourteen Bravais lattices can be formed by interference of four noncoplanar beams. Opt. Lett. 2002, 27, 900-902.

13. Turberfield, A.J. Photonic crystals made by holographic lithography. MRS Bull. 2001, 26, 632-636.

14. Mei, D.; Cheng, B.; Hu, W.; Li, Z.; Zhang, D. Three-dimensional ordered patterns by light interference. Opt. Lett. 1995, 20, 429-431.

15. Petsas, A.I.; Coates, A.B.; Grynberg, G. Crystallography of optical lattices. Phys. Rev. A 1994, 50, 5173-5189.

16. Ono, Y.; Ochi, T. All fourteen Bravais lattices can be fabricated by triple exposure of two-beam interference fringes. Proc. SPIE 2006, 6327, 632709.

17. Dwivedi, A.; Xavier, J.; Joseph, J.; Singh, K. Formation of all fourteen Bravais lattices of three-dimensional photonic crystal structures by a dual beam multiple-exposure holographic technique. Appl. Opt. 2008, 47, 1973-1980.

18. Wu, L.J.; Zhong, Y.C.; Wong, K.S.; Wang, G.P.; Yuan, L. Fabrication of hetero-binary and honeycomb photonic crystals by one-step holographic lithography. Appl. Phys. Lett. 2006, 88, 09115.

19. Solak, H.H. Space-invariant multiple-beam achromatic EUV interference lithography. Microelectron. Eng. 2005, 78-79, 410-416.

20. Jimenez-Ceniceros, A.; Trejo-Duran, M.; Alvarado-Mendez, E.; Castano, V.M. Extinction zones and scalability in N-beam interference lattices. Opt. Commun. 2010, 283, 362-367.

21. Mao, W.D.; Dong, J.W.; Zhong, Y.C.; Liang, G.Q.; Wang, H.Z. Formation principles of two-dimensional compound photonic lattices by one-step holographic lithography. Opt. Express 2005, 13, 2994-2999. 
22. Chan, T.Y.M.; Toader, O.; John, S. Photonic band gap templating using optical interference lithography. Phys. Rev. E 2005, 71, 046605.

23. Dedman, E.R.; Sharp, D.N.; Turberfield, A.J.; Blanford, C.F.; Denning, R.G. Photonic crystals with a chiral basis by holographic lithography. Photonics Nanostruct. 2005, 3, 79-83.

24. Lai, N.D.; Lin, J.H.; Hsu, C.C. Fabrication of highly rotational symmetric quasi-periodic structures by multiexposure of a three-beam interference technique. Appl. Opt. 2007, 46, 5645-5648.

25. Juodkazis, S.; Mizeikis, V.; Misawa, H. Three-dimensional microfabrication of materials by femtosecond lasers for photonics applications. J. Appl. Phys. 2009, 106, 051101.

26. Zaccaria, R.P.; Shoji, S.; Sun, H.B.; Kawata, S. Multi-shot interference approach for any kind of Bravais lattice. Appl. Phys. B 2008, 93, 251-256.

27. Tam, W.Y. Icosahedral quasicrystals by optical interference holography. Appl. Phys. Lett. 2006, 89, 251111.

28. Stay, J.L.; Gaylord, T.K. Conditions for primitive-lattice-vector-direction equal contrasts in four-beam-interference lithography. Appl. Opt. 2009, 48, 4801-4813.

29. Ullal, C.K.; Maldovan, M.; Wohlgemuth, M.; Thomas, E.L.; White, C.A.; Yang, S. Triply periodic bicontinuous structures through interference lithography: A level-set approach. J. Opt. Soc. Am. A 2003, 20, 948-954.

30. Yang, X.L.; Cai, L.Z.; Wang, Y.R.; Dong, G.Y.; Shen, X.X.; Meng, X.F.; Hu, Y. Large complete bandgaps in a two-dimensional square photonic crystal with isolated single-atom dielectric rods in air. Nanotechnology 2008, 19, 025201-025201.

31. Moon, J.H.; Pine, D.J.; Yang, S.-M. Fabrication of two-dimensional photonic crystals of non-spherical atoms by holographic lithography. In Proceedings of Photonic Crystal Materials and Nanostructures, Strasbourg, France, 27-29 April 2004; pp. 95-99.

32. Quinonez, F.; Menezes, J.W.; Cescato, L.; Rodriguez-Esquerre, V.F.; Hernandez-Figueroa, H.; Mansano, R.D. Band gap of hexagonal 2D photonic crystals with elliptical holes recorded by interference lithography. Opt. Express 2006, 14, 4873-4879.

33. Hung, Y., Jr.; Lee, S.-L.; Pan, Y.-T. Photonic bandgap analysis of photonic crystal slabs with elliptical holes and their formation with laser holography. J. Opt. 2010, 12, 015102.

34. Ao, X.; He, S. Two-stage design method for realization of photonic bandgap structures with desired symmetries by interference lithography. Opt. Express 2004, 12, 978-983.

35. Chanda, D.; Abolghasemi, L.; Herman, P.R. Numerical band calculation of holographically formed periodic structures with irregular motif. Proc. SPIE 2006, 6128, 61281E.

36. Qiu, M.; He, S. Optimal design of a two-dimensional photonic crystal of square lattice with a large complete two-dimensional bandgap. J. Opt. Soc. Am. B 2000, 17, 1027-1030.

37. Qi, L.M.; Yang, Z.Q.; Gao, X.; Liu, W.X.; Liang, Z. Research on three types of rhombus lattice photonic band structures. J. Electromagn. Waves Appl. 2008, 22, 1155-1164.

38. Pu, Y.-Y.; Liang, G.-Q.; Mao, W.-D.; Dong, J.-W.; Wang, H.-Z. Fabrication of two-dimensional photonic crystals with triangular rods by single-exposure holographic lithography. Chin. Phys. Lett. 2007, 24, 983-985. 
39. Cai, L.Z.; Dong, G.Y.; Feng, C.S.; Yang, X.L.; Shen, X.X.; Meng, X.F. Holographic design of a two-dimensional photonic crystal of square lattice with a large two-dimensional complete bandgap. J. Opt. Soc. Am. B 2006, 23, 1708-1711.

40. Wang, R.; Wang, X.-H.; Gu, B.-Y.; Yang, G.-Z. Effects of shapes and orientations of scatterers and lattice symmetries on the photonic band gap in two-dimensional photonic crystals. J. Appl. Phys. 2001, 90, 4307-4313.

41. Cai, L.Z.; Shen, X.X.; Yang, X.L.; Dong, G.Y.; Meng, X.F.; Xu, X.F.; Wang, Y.R. Holographic design of hexagonal photonic crystals of irregular columns with large full band gap. Opt. Commun. 2006, 267, 305-309.

42. Yang, X.L.; Cai, L.Z.; Wang, Y.R.; Feng, C.S.; Dong, G.Y.; Shen, X.X.; Meng, X.F.; Hu, Y. Optimization of band gap of photonic crystals fabricated by holographic lithography. Europhys. Lett. 2008, 81, 14001.

43. Chien, C.W.; Lee, Y.C.; Lee, P.S.; Chang, J.Y.; Chen, J.C. Analysis of a two-dimensional photonic bandgap structure fabricated by an interferometric lithographic system. Appl. Opt. 2007, 46, 3196-3204.

44. Ohlinger, K.; Lin, Y.K.; Qualls, J.S. Maximum and overlapped photonic band gaps in both transverse electric and transverse magnetic polarizations in two-dimensional photonic crystals with low symmetry. J. Appl. Phys. 2009, 106, 063520.

45. Li, Z.-Y.; Wang, J.; Gu, B.Y. Full band gap in fcc and bec photonic band gaps structure: Non-spherical atom. J. Phys. Soc. Jpn. 1998, 67, 3288-3291.

46. Kang, H.K.; Lee, K.H.; Wong, C.C.; Romanato, F. ID to 2D transitional structure of plasmonic crystals: fabrication and characterization. Appl. Phys. B 2009, 97, 671-677.

47. Noda, S. Photonic crystal lasers-ultimate nanolasers and broad-area coherent lasers. J. Opt. Soc. Am. B Opt. Phys. 2010, 27, B1-B8.

48. Noda, S.; Yokoyama, M.; Imada, M.; Chutinan, A.; Mochizuki, M. Polarization mode control of two-dimensional photonic crystal laser by unit cell structure design. Science 2001, 293, 1123-1125.

49. Hu, B.; Lu, M.; Li, W.; Zou, K.; Zhou, Z.; Lin, A.; Li, N. High birefringent rhombic-hole photonic crystal fibers. Appl. Opt. 2010, 49, 6098-6101.

50. Jang, J.; Jhaveri, S.J.; Rasin, B.; Koh, C.; Ober, C.K.; Thomas, E.L. Three-dimensionally-patterned submicrometer-scale hydrogel/air networks that offer a new platform for biomedical applications. Nano Lett. 2008, 8, 1456-1460.

51. Huang, J.; Beckemper, S.; Gillner, A.; Wang, K. Tunable surface texturing by polarizationcontrolled three-beam interference. J. Micromech. Microeng. 2010, 20, 095004.

52. Vavassori, P.; Donzelli, O.; Metlushko, V.; Grimsditch, M.; Ilic, B.; Neuzil, P.; Kumar, R. Magnetic switching in submicron-scale periodic magnetic arrays. J. Appl. Phys. 2000, 88, 999-1003.

53. Tang, Z.X.; Peng, R.W.; Fan, D.Y.; Wen, S.C.; Zhang, H.; Qian, L.J. Absolute left-handed behaviors in a triangular elliptical-rod photonic crystal. Opt. Express 2005, 13, 9796-9803.

54. Shen, X.X.; Yang, X.L.; Cai, L.Z.; Dong, G.Y.; Meng, X.F.; Xu, X.F.; Wang, Y.R. Negative refractions in two-dimensional photonic crystals formed by holographic lithography. Opt. Express 2007, 15, 8003-8009. 
55. Yuan, L.; Wang, G.P.; Huang, X.K. Arrangements of four beams for any Bravais lattice. Opt. Lett. 2003, 28, 1769-1771.

56. Li, E.B.; Xi, J.T.; Chicharo, J. Predication of multi-dimensional photonic crystal structures generated by multi-beam interference in holographic lithography. Smart Mater. Struct. 2006, 15, 158-164.

57. Do, D.B.; Lai, N.D.; Wu, C.Y.; Lin, J.H.; Hsu, C.C. Fabrication of ellipticity-controlled microlens arrays by controlling the parameters of the multiple-exposure two-beam interference technique. Appl. Opt. 2011, 50, 579-585.

58. Menezes, J.W.; Cescato, L. Recording different geometries of 2D hexagonal photonic crystals by choosing the phase between two-beam interference exposures. Opt. Express 2006, 14, 8578-8583.

59. Burrow, G.M.; Gaylord, T.K. Constrained parametric optimization of point geometries in multi-beam-interference lithography. In Proceedings of Frontiers in Optics 2010, Rochester, NY, USA, 27 October 2010.

60. Yang, X.L.; Cai, L.Z.; Liu, Q. Polarization optimization in the interference of four umbrellalike symmetric beams for making three-dimensional periodic microstructures. Appl. Opt. 2002, 41, 6894-6900.

61. Su, H.M.; Zhong, Y.C.; Wang, X.; Zheng, X.G.; Xu, J.F.; Wang, H.Z. Effects of polarization on laser holography for microstructure fabrication. Phys. Rev. E 2003, 67, 056619.

62. Fernandez, A.; Decker, J.Y.; Herman, S.M.; Phillion, D.W.; Sweeney, D.W.; Perry, M.D. Methods for fabricating arrays of holes using interference lithography. J. Vac. Sci. Technol. B 1997, 15, 2439-2443.

63. Fernandez, A.; Phillion, D.W. Effects of phase shifts on four-beam interference patterns. Appl. Opt. 1998, 37, 473-478.

64. Ellman, M.; Rodriguez, A.; Perez, N.; Echeverria, M.; Verevkin, Y.K.; Peng, C.S.; Berthou, T.; Wang, Z.; Olaizola, S.M.; Ayerdi, I. High-power laser interference lithography process on photoresist: Effect of laser fluence and polarisation. Appl. Surf. Sci. 2009, 255, 5537-5541.

65. Stay, J.L.; Gaylord, T.K. Contrast in four-beam-interference lithography. Opt. Lett. 2008, 33, 1434-1436.

66. Chanda, D.; Abolghasemi, L.E.; Herman, P.R. Single laser exposure fabrication of diamond-like 3-dimensional photonic crystal microstructures using circularly polarized light. Appl. Phys. A 2008, 93, 33-37.

67. Meisel, D.C.; Wegener, M.; Busch, K. Three-dimensional photonic crystals by holographic lithography using the umbrella configuration: symmetries and complete photonic band gaps. Phys. Rev. B 2004, 70, 165104.

68. Cai, L.Z.; Yang, X.L.; Wang, Y.R. Interference of three noncoplanar beams: Patterns, contrast and polarization optimization. J. Mod. Opt. 2002, 49, 1663-1672.

69. Cai, L.Z.; Yang, X.L. Interference of circularly polarized light: contrast and application in fabrication of three-dimensional periodic microstructures. Opt. Laser Technol. 2002, 34, 671-674.

70. Yang, X.L.; Cai, L.Z. Wave design of the interference of three noncoplanar beams for microfiber fabrication. Opt. Commun. 2002, 208, 293-297. 
71. Yang, X.L.; Cai, L.Z. Wave design and polarization optimization in the interference of four non-coplanar beams for making three-dimensional periodical microstructures. J. Mod. Opt. 2003, 50, 1445-1453.

72. Yang, X.L.; Cai, L.Z.; Wang, Y.R.; Liu, Q. Interference of four umbrellalike beams by a diffractive beam splitter for fabrication of two-dimensional square and trigonal lattices. Opt. Lett. 2003, 28, 453-455.

73. Stay, J.L.; Gaylord, T.K. Three-beam-interference lithography: Contrast and crystallography. Appl. Opt. 2008, 47, 3221-3230.

74. Stay, J.L. Multi-Beam-Interference-Based Methodology for the Fabrication of Photonic Crystal Structures. Ph.D. Thesis, Electrical and Computer Engineering, Georgia Institute of Technology, Atlanta, GA, USA, 2009.

75. Salaun, M.; Audier, M.; Duneau, M.; Delyon, F. Holographic lithography of a two-dimensional hexagonal structure: effect of beam polarization. Appl. Surf. Sci. 2007, 254, 850-854.

76. Lasagni, A.F.; Dajun, Y.; Das, S. Layer-by-layer interference lithography of three-dimensional microstructures in SU-8. Adv. Eng. Mater. 2009, 11, 408-411.

77. Pang, L.; Nakagawa, W.; Fainman, Y. Fabrication of two-dimensional photonic crystals with controlled defects by use of multiple exposures and direct write. Appl. Opt. 2003, 42, 5450-5456.

78. Pati, G.S.; Heilmann, R.K.; Konkola, P.T.; Joo, C.; Chen, C.G.; Murphy, E.; Schattenburg, M.L. Generalized scanning beam interference lithography system for patterning gratings with variable period progressions. J. Vac. Sci. Technol. B 2002, 20, 2617-2621.

79. Gutierrez-Rivera, L.E.; Cescato, L. SU-8 submicrometric sieves recorded by UV interference lithography. J. Micromech. Microeng. 2008, 18, 115003.

80. Gauthier, R.C.; Mnaymneh, K.W. Design of photonic band gap structures through a dual-beam multiple exposure technique. Opt. Laser Technol. 2004, 36, 625-633.

81. Moormann, C.; Bolten, J.; Kurz, H. Spatial phase-locked combination lithography for photonic crystal devices. In Proceedings of 29th International Conference on Micro and Nano Engineering, Delft, The Netherlands, 22-25 September 2003; pp. 417-422.

82. Lee, F.Y.; Fung, K.H.; Tang, T.L.; Tam, W.Y.; Chan, C.T. Fabrication of gold nano-particle arrays using two-dimensional templates from holographic lithography. Curr. Appl. Phys. 2009, 9 , 820-825.

83. Dyachenko, P.N.; Karpeev, S.V.; Fesik, E.V.; Miklyaev, Y.V.; Pavelyev, V.S.; Malchikov, G.D. The three-dimensional photonic crystals coated by gold nanoparticles. Opt. Commun. 2011, 284, 885-888.

84. MacLeod, B.D.; Kelsey, A.F.; Leclerc, M.A.; Resler, D.P.; Liberman, S.; Nole, J.P. Fully automated interference lithography. In Proceedings of Emerging Lithographic Technologies VI, Santa Clara, CA, USA, 5-7 March 2002; pp. 910-921.

85. Rodriguez, A.; Echeverria, M.; Ellman, M.; Perez, N.; Verevkin, Y.K.; Peng, C.S.; Berthou, T.; Zuobin, W.; Ayerdi, I.; Savall, J.; Olaizola, S.M. Laser interference lithography for nanoscale structuring of materials: From laboratory to industry. Microelectron. Eng. 2009, 86, 937-940.

86. Lu, C.; Hu, X.K.; Dimov, S.S.; Lipson, R.H. Controlling large-scale film morphology by phase manipulation in interference lithography. Appl. Opt. 2007, 46, 7202-7206. 
87. Xie, X.S.; Li, M.; Guo, J.; Liang, B.; Wang, Z.X.; Sinitskii, A.; Xiang, Y.; Zhou, J.Y. Phase manipulated multi-beam holographic lithography for tunable optical lattices. Opt. Express 2007, 15, 7032-7037.

88. Kondo, T.; Matsuo, S.; Juodkazis, S.; Misawa, H. Femtosecond laser interference technique with diffractive beam splitter for fabrication of three-dimensional photonic crystals. Appl. Phys. Lett. 2001, 79, 725-727.

89. Pissadakis, S.; Pappas, C. Planar periodic structures fabricated in Er/Yb-codoped phosphate glass using multi-beam ultraviolet laser holography. Opt. Express 2007, 15, 4296-4303.

90. Levine, Z.H.; Grantham, S.; Lucatorto, T.B. Design considerations for a cascaded grating interferometer suitable for extreme ultraviolet interference lithography. J. Microlith. Microfab. Microsys. 2009, 8, 021202.

91. Klein-Wiele, J.H.; Simon, P. Fabrication of periodic nanostructures by phase-controlled multiple-beam interference. Appl. Phys. Lett. 2003, 83, 4707-4709.

92. Savas, T.A.; Shah, S.N.; Schattenburg, M.L.; Carter, J.M.; Smith, H.I. Achromatic interferometric lithography for 100-nm-period gratings and grids. J. Vac. Sci. Technol. B 1995, 13, 2732-2735.

93. Cai, L.Z.; Yang, X.L.; Liu, Q.; Wang, Y.R. What kind of Bravais lattices can be made by the interference of four umbrellalike beams? Opt. Commun. 2003, 224, 243-246.

94. Yang, X.L.; Cai, L.Z.; Wang, Y.R.; Liu, Q. Interference technique by three equal-intensity umbrellalike beams with a diffractive beam splitter for fabrication of two-dimensional trigonal and square lattices. Opt. Commun. 2003, 218, 325-332.

95. Tam, W.Y. Woodpile and diamond structures by optical interference holography. J. Opt. A Pure Appl. Opt. 2007, 9, 1076-1081.

96. Dong, G.Y.; Cai, L.Z.; Yang, X.L.; Shen, X.X.; Meng, X.F.; Xu, X.F.; Wang, Y.R. Holographic design and band gap evolution of photonic crystals formed with five-beam symmetric umbrella configuration. Opt. Express 2006, 14, 8096-8102.

97. Pang, Y.K.; Lee, J.C.W.; Ho, C.T.; Tam, W.Y. Realization of woodpile structure using optical interference holography. Opt. Express 2006, 14, 9113-9119.

98. Brundrett, D.L.; Gaylord, T.K.; Glytsis, E.N. Polarizing mirror/absorber for visible wavelengths based on a silicon subwavelength grating: Design and fabrication. Appl. Opt. 1998, 37, 2534-2541.

99. Stay, J.L.; Burrow, G.M.; Gaylord, T.K. Three-beam interference lithography methodology. Rev. Sci. Instrum. 2011, 82, 023115.

100. Mai, X.; Moshrefzadeh, R.; Gibson, U.J.; Stegeman, G.I.; Seaton, C.T. Simple versatile method for fabricating guided-wave gratings. Appl. Opt. 1985, 24, 3155-3161.

101. Guan, Y.; Pedraza, A.J. Synthesis and characterization of self-organized nanostructure arrays generated by laser irradiation. Mat. Res. Soc. Symp. Proc. 2004, 818, 335-340.

102. de Boor, J.; Geyer, N.; Gosele, U.; Schmidt, V. Three-beam interference lithography: Upgrading a Lloyd's interferometer for single-exposure hexagonal patterning. Opt. Lett. 2009, 34, 1783-1785.

103. de Boor, J.; Dong Sik, K.; Schmidt, V. Sub-50 nm patterning by immersion interference lithography using a Littrow prism as a Lloyd's interferometer. Opt. Lett. 2010, 35, 3450-3452. 
104. Rizvi, N.H.; Gower, M.C. Production of submicrometer period Bragg gratings in optical fibers using wavefront division with a biprism and an excimer laser source. Appl. Phys. Lett. 1995, 67, 739-741.

105. Xiang, W.; Liang, J.; Zhang, G.; Wu, L.; Wong, K.S.; Wong, G.K.L. Non-coplanar multi-beam interference produced by one triangular pyramid for fabricating photonic crystals. Chin. Opt. Lett. 2005, 3, 712-714.

106. Zhong, Y.C.; Wu, L.J.; Su, H.M.; Wong, K.S.; Wang, H.Z. Fabrication of photonic crystals with tunable surface orientation by holographic lithography. Opt. Express 2006, 14, 6837-6843.

107. Lei, M.; Yao, B.; Rupp, R.A. Structuring by multi-beam interference using symmetric pyramids. Opt. Express 2006, 14, 5803-5811.

108. Miklyaev, Y.V.; Meisel, D.C.; Blanco, A.; von Freymann, G.; Busch, K.; Koch, W.; Enkrich, C.; Deubel, M.; Wegener, M. Three-dimensional face-centered-cubic photonic crystal templates by laser holography: Fabrication, optical characterization, and band-structure calculations. Appl. Phys. Lett. 2003, 82, 1284-1286.

109. Ullal, C.K.; Maldovan, M.; Thomas, E.L.; Chen, G.; Han, Y.J.; Yang, S. Photonic crystals through holographic lithography: Simple cubic, diamond-like, and gyroid-like structures. Appl. Phys. Lett. 2004, 84, 5434-5436.

110. Lin, C.H.; Zhu, Z.H.; Lo, Y.H. New grating fabrication technology for optoelectronic devices: Cascaded self-induced holography. Appl. Phys. Lett. 1995, 67, 3072-3074.

111. Berger, V.; Gauthier-Lafaye, O.; Costard, E. Photonic band gaps and holography. J. Appl. Phys. 1997, 82, 60-64.

112. Divliansky, I.B.; Shishido, A.; Khoo, I.C.; Mayer, T.S.; Pena, D.; Nishimura, S.; Keating, C.D.; Mallouk, T.E. Fabrication of two-dimensional photonic crystals using interference lithography and electrodeposition of CdSe. Appl. Phys. Lett. 2001, 79, 3392-3394.

113. Lin, Y.; Herman, P.R. Effect of structural variation on the photonic band gap in woodpile photonic crystal with body-centered-cubic symmetry. J. Appl. Phys. 2005, 98, 63104-63101.

114. Schneider, G.J.; Wetzel, E.D.; Murakowski, J.A.; Prather, D.W. Fabrication of three-dimensional "Yablonovite" photonic crystals by multiple-exposure UV interference lithography. In Proceedings of Micromachining Technology for Micro-Optics and Nano-Optics III, San Jose, CA, USA, 25-27 January 2005; pp. 9-17.

115. Chelnokov, A.; Rowson, S.; Lourtioz, J.M.; Berger, V.; Courtois, J.Y. An optical drill for the fabrication of photonic crystals. J. Opt. A Pure Appl. Opt. 1999, 1, 3-6.

116. Divliansky, I.; Mayer, T.S.; Holliday, K.S.; Crespi, V.H. Fabrication of three-dimensional polymer photonic crystal structures using single diffraction element interference lithography. Appl. Phys. Lett. 2003, 82, 1667-1669.

117. Okai, M.; Tsuji, S.; Chinone, N.; Harada, T. Novel method to fabricate corrugation for a lambda/4-shifted distributed feedback laser using a grating photomask. Appl. Phys. Lett. 1989, 55, 415-417.

118. Jeon, S.; Park, J.; Cirelli, R.; Yang, S.; Heitzman, C.; Braun, P.; Kenis, P.; Rogers, J. Fabricating complex three-dimensional nanostructures with high-resolution conformable phase masks. PNAS 2004, 101, 12428-12433. 
119. Lin, Y.; Herman, P.R.; Abolghasemi, E.L. Proposed single-exposure holographic fabrication of microsphere-type photonic crystals through phase-mask techniques. J. Appl. Phys. 2005, 97, 096102.

120. Lin, Y.; Herman, P.R.; Darmawikarta, K. Design and holographic fabrication of tetragonal and cubic photonic crystals with phase mask: Toward the mass-production of three-dimensional photonic crystals. Appl. Phys. Lett. 2005, 86, 071117.

121. Hill, K.O.; Malo, B.; Bilodeau, F.; Johnson, D.C.; Albert, J. Bragg gratings fabricated in monomode photosensitive optical fiber by UV exposure through a phase mask. Appl. Phys. Lett. 1993, 62, 1035-1037.

122. Chanda, D.; Abolghasemi, L.; Herman, P.R. One-dimensional diffractive optical element based fabrication and spectral characterization of three-dimensional photonic crystal templates. Opt. Express 2006, 14, 8568-8577.

123. Lin, Y.; Rivera, D.; Chen, K.P. Woodpile-type photonic crystals with orthorhombic or tetragonal symmetry formed through phase mask techniques. Opt. Express 2006, 14, 887-892.

124. Chan, T.Y.M.; Toader, O.; John, S. Photonic band-gap formation by optical-phase-mask lithography. Phys. Rev. E 2006, 73, 46610-46611.

125. Chanda, D.; Herman, P.R. Two-dimensional diffractive optical element based fabrication of 3D photonic crystal templates. Proc. SPIE 2007, 6480, 64800Q.

126. Lin, Y.; Harb, A.; Rodriguez, D.; Lozano, K.; Xu, D.; Chen, K.P. Holographic fabrication of photonic crystals using multidimensional phase masks. J. Appl. Phys. 2008, 104, 113111.

127. Zhou, G.; Chau, F.S. Three-dimensional photonic crystal by holographic contact lithography using a single diffraction mask. Appl. Phys. Lett. 2007, 90, 181106-181101.

128. Di, X.; Chen, K.P.; Ohlinger, K.; Lin, Y. Holographic fabrication of diamondlike photonic crystal template using two-dimensional diffractive optical elements. Appl. Phys. Lett. 2008, 93, 031101.

129. George, M.C.; Nelson, E.C.; Rogers, J.A.; Braun, P.V. Direct fabrication of 3D periodic inorganic microstructures using conformal phase masks. Angew. Chem. Int. Edit. 2008, 48, 144-148.

130. Chanda, D.; Zachari, N.; Haque, M.; Ng, M.L.; Herman, P.R. Flexible fabrication of three-dimensional optical-domain photonic crystals using a combination of single-laser-exposure diffractive-optics lithography and template inversion. Opt. Lett. 2009, 34, 3920-3922.

131. Brueck, S.R.J. Optical and interferometric lithography-Nanotechnology enablers. Proc. IEEE 2005, 93, 1704-1721.

132. Horner, J.L. Additional property of interferometer symmetry. Appl. Opt. 1978, 17, 505-505.

133. Lai, N.D.; Liang, W.P.; Lin, J.H.; Hsu, C.C.; Lin, C.H. Fabrication of two- and three-dimensional periodic structures by multi-exposure of two-beam interference technique. Opt. Express 2005, 13, 9605-9611.

134. Divliansky, I.; Mayer, T.S. Three-dimensional low-index-contrast photonic crystals fabricated using a tunable beam splitter. Nanotechnol. 2006, 17, 1241.

135. Xu, J.; Ma, R.; Wang, X.; Tam, W.Y. Icosahedral quasicrystals for visible wavelengths by optical interference holography. Opt. Express 2007, 15, 4287-4295. 
136. Wu, L.; Zhong, Y.; Chan, C.T.; Wong, K.S.; Wang, G.P. Fabrication of large area two- and three-dimensional polymer photonic crystals using single refracting prism holographic lithography. Appl. Phys. Lett. 2005, 86, 241102.

137. Dakss, M.L.; Kuhn, L.; Heidrich, P.F.; Scott, B.A. Grating coupler for efficient excitation of optical guided waves in thin films. Appl. Phys. Lett. 1970, 16, 523-525.

138. Zaidi, S.H.; Brueck, S.R.J. Multiple-exposure interferometric lithography. J. Vac. Sci. Technol. B 1993, 11, 658-666.

139. Lim, K.-S.; Lee, M.; Shin, H.; Kim, H. Step-wise Ag thin film patterns fabricated by holographic lithography. Thin Solid Films 2009, 517, 3273-3275.

140. Kondo, T.; Juodkazis, S.; Misawa, H. Reduction of capillary force for high-aspect ratio nanofabrication. Appl. Phys. A 2005, A81, 1583-1586.

141. Amako, J.; Sawaki, D.; Kato, M. Fringe-shifting interferometric laser lithography with optical nonlinearity for micro- and nanofabrications. Appl. Phys. Lett. 2007, 91, 054105.

142. Lasagni, A.; Dajun, Y.; Peng, S.; Das, S. Rapid fabrication of biocompatible hydrogels microdevices using laser interference lithography. Proc. SPIE 2009, 7365, 73650I.

143. Zhong, Y.; Zhou, J.; Wong, K.S. Two-photon fabrication of photonic crystals by single-beam laser holographic lithography. J. Appl. Phys. 2010, 107, 074311.

144. Yang, Y.; Wang, G.P. Realization of periodic and quasiperiodic microstructures with sub-diffraction-limit feature sizes by far-field holographic lithography. Appl. Phys. Lett. 2006, 89, 111104-111101.

145. Zhu, T.-F.; Tan, B.-H.; Pan, X.-F.; Tao, W.-D. Fabrication of two-and three-dimensional periodic submicron structures by holographic lithography with a $635 \mathrm{~nm}$ laser and matched photopolymer. Chin. Phys. B 2010, 19, 014218.

146. Arsh, A.; Klebanov, M.; Lyubin, V.; Shapiro, L.; Feigel, A.; Veinger, M.; Sfez, B. Glassy $\mathrm{mAs}_{2} \mathrm{~S}_{3} \mathrm{nAs}_{2} \mathrm{Se}_{3}$ photoresist films for interference laser lithography. Opt. Mater. 2004, 26, 301-304.

147. Wang, X.; Su, H.; Zhang, L.; He, Y.; Zheng, X.; Wang, H. Fabrication of a submicrometer crystalline structure by thermoplastic holography. Appl. Opt. 2001, 40, 5588-5591.

148. Yang, S.; Megens, M.; Aizenberg, J.; Wiltzius, P.; Chaikin, P.M.; Russel, W.B. Creating periodic three-dimensional structures by multibeam interference of visible laser. Chem. Mater. 2002, 14, 2831-2833.

149. Aydin, C.; Zaslavsky, A.; Sonek, G.J.; Goldstein, J. Reduction of reflection losses in $\mathrm{ZnGeP}_{2}$ using motheye antireflection surface relief structures. Appl. Phys. Lett. 2002, 80, 2242-2244.

150. Liu, T.; Fallahi, M.; Moloney, J.V.; Mansuripur, M. Fabrication of two-dimensional photonic crystals with embedded defects using blue-laser-writer and optical holography. IEEE Phot. Tech. L. 2006, 18, 1100-1102.

151. Berendsen, C.W.J.; Skeren, M.; Najdek, D.; Cerny, F. Superhydrophobic surface structures in thermoplastic polymers by interference lithography and thermal imprinting. Appl. Surf. Sci. 2009, 255, 9305-9310.

152. Fucetola, C.P.; Korre, H.; Berggren, K.K. Low-cost interference lithography. J. Vac. Sci. Technol. B 2009, 27, 2958-2961. 
153. Jang, J.-H.; Dendukuri, D.; Alan, H.T.; Thomas, E.L.; Doyle, P.S. A route to three-dimensional structures in a microfluidic device: Stop-flow interference lithography. Angew. Chem. Int. Edit. 2007, 46, 9027-9031.

154. Suleski, T.J.; Baggett, B.; Delaney, W.F.; Koehler, C.; Johnson, E.G. Fabrication of high-spatial-frequency gratings through computer-generated near-field holography. Opt. Lett. 1999, 24, 602-604.

155. de Ridder, R.M.; Bostan, C.G.; van Wolferen, H.A.G.M.; van Dorssen, I.; Vogelaar, L.; Segerink, F.B.; Kuipers, L.; van Hulst, N.F. Fabrication of photonic crystal slabs and defects using laser interference lithography and focused ion beam-assisted deposition. In Proceedings of the 4th International Conference on Transparent Optical Networks, Warsaw, Poland, 21-25 April 2002; pp. 14-19

156. Chen, X.; Zaidi, S.H.; Brueck, S.R.J.; Devine, D.J. Interferometric lithography of sub-micrometer sparse hole arrays for field-emission display applications. J. Vac. Sci. Technol. B 1996, 14, 3339-3349.

157. Campbell, M.; Sharp, D.N.; Harrison, M.T.; Denning, R.G.; Turberfield, A.J. Fabrication of photonic crystals for the visible spectrum by holographic lithography. Nature 2000, 404, 53-56.

158. Berger, V.; Gauthier-Lafaye, O.; Costard, E. Fabrication of a $2 \mathrm{D}$ photonic bandgap by a holographic method. Electron. Lett. 1997, 33, 425-426.

159. Lai, N.D.; Liang, W.P.; Lin, J.H.; Hsu, C.C. Rapid fabrication of large-area periodic structures containing well-defined defects by combining holography and mask techniques. Opt. Express 2005, 13, 5331-5337.

160. Peng, C.S.; Tan, C. Fast, high efficiency and cost-effective laser nano-lithography. In Proceedings of 5th International Symposium on Advanced Optical Manufacturing and Testing Technologies: Design, Manufacturing, and Testing of Micro- and Nano-Optical Devices and Systems, Dalian, China, 26-29 April 2010; pp. 765708-765712.

161. Lemme, M.C.; Moormann, C.; Lerch, H.; Moller, M.; Vratzov, B.; Kurz, H. Triple-gate metal-oxide-semiconductor field effect transistors fabricated with interference lithography. Nanotechnology 2004, 15, 208-210.

162. Ura, S.; Hamada, M.; Ohmori, J.; Nishio, K.; Kintaka, K. Free-space-wave drop demultiplexing waveguide device fabricated by use of the interference exposure method. Appl. Opt. 2006, 45, 22-26.

163. Fritze, M.; Bloomstein, T.M.; Tyrrell, B.; Fedynyshyn, T.H.; Efremow, N.N., Jr,; Hardy, D.E.; Cann, S.; Lennon, D.; Spector, S.; Rothschild, M.; Brooker, P. Hybrid optical maskless lithography: scaling beyond the $45 \mathrm{~nm}$ node. J. Vac. Sci. Technol. B 2005, 23, 2743-2748.

164. Capeluto, M.G.; Vaschenko, G.; Grisham, M.; Marconi, M.C.; Luduena, S.; Pietrasanta, L.; Yunfeng, L.; Parkinson, B.; Menoni, C.S.; Rocca, J.J. Nanopatterning with interferometric lithography using a compact lambda = 46.9-nm laser. IEEE Nanotechnol. 2006, 5, 3-7.

165. Ritucci, A.; Reale, A.; Zuppella, P.; Reale, L.; Tucceri, P.; Tomassetti, G.; Bettotti, P.; Pavesi, L. Interference lithography by a soft X-ray laser beam: nanopatterning on photoresists. J. Appl. Phys. 2007, 102, 034313. 
166. Solak, H.H.; He, D.; Li, W.; Singh-Gasson, S.; Cerrina, F.; Sohn, B.H.; Yang, X.M.; Nealey, P. Exposure of $38 \mathrm{~nm}$ period grating patterns with extreme ultraviolet interferometric lithography. Appl. Phys. Lett. 1999, 75, 2328-2330.

167. Isoyan, A.; Cheng, Y.C.; Jiang, F.; Wallace, J.; Cerrina, F.; Bollepalli, S. Progress in extreme ultraviolet interferometric and holographic lithography. J. Vac. Sci. Technol. B 2007, 25, 2145-2150.

168. Gates, B.D.; Xu, Q.; Stewart, M.; Ryan, D.; Willson, C.G.; Whitesides, G.M. New Approaches to nanofabrication: molding, printing, and other techniques. Chem. Rev. 2005, 105, 1171-1196.

169. Sreekanth, K.V.; Chua, J.K.; Murukeshan, V.M. Interferometric lithography for nanoscale feature patterning: A comparative analysis between laser interference, evanescent wave interference, and surface plasmon interference. Appl. Opt. 2010, 49, 6710-6717.

170. Luttge, R. Massively parallel fabrication of repetitive nanostructures: nanolithography for nanoarrays. J. Phys. D Appl. Phys. 2009, 42, 123001.

171. Smith, H.I. Low cost nanolithography with nanoaccuracy. In Proceedings of Rutherford Memorial Workshop on Semiconductor Nanostructures, Enschede, The Netherlands, 5-9 February 2001; pp. 104-109.

172. Greenway, R.T.; Hendel, R.; Jeong, K.; Kahng, A.B.; Petersen, J.S.; Rao, Z.; Smayling, M.C. Interference assisted lithography for patterning of 1D gridded design. Proc. SPIE 2009, 7271, $72712 \mathrm{U}$.

173. Lin, Y.K.; Harb, A.; Lozano, K.; Xu, D.; Chen, K.P. Five beam holographic lithography for simultaneous fabrication of three dimensional photonic crystal templates and line defects using phase tunable diffractive optical element. Opt. Express 2009, 17, 16625-16631.

174. Murakowski, J.; Schneider, G.J.; Prather, D. Fabrication of 3-dimensional Photonic Crystals with Embedded Defects. In Micromachining Technology for Micro-Optics and Nano-Optics II, San Jose, CA, USA, 27-29 January 2004; pp. 181-189.

175. Zhao, L.; Xuan, Y.; Qi, M. Generating integrated-circuit patterns via cutting and stitching of gratings. J. Vac. Sci. Technol. B 2009, 27, 2750-2754.

176. Szymanski, D.; Dylewicz, R.; Patela, S.; Bartkiewicz, S.; Miniewicz, A. Defects generation in photonic crystal pattern by electron beam induced deposition technique. Proc. SPIE 2005, 5950, $59501 \mathrm{~T}$.

177. van Rijn, C.J.M. Laser interference as a lithographic nanopatterning tool. J. Microlith. Microfab. Microsys. 2006, 5, 11012-11011.

178. Sun, H.-B.; Nakamura, A.; Kaneko, K.; Shoji, S.; Kawata, S. Direct laser writing defects in holographic lithography-created photonic lattices. Opt. Lett. 2005, 30, 881-883.

179. Braun, P.V.; Rinne, S.A.; Garcia-Santamaria, F. Introducing defects in 3D photonic crystals: State of the art. Adv. Mater. 2006, 18, 2665-2678.

180. Parisse, P.; Luciani, D.; D’Angelo, A.; Santucci, S.; Zuppella, P.; Tucceri, P.; Reale, A.; Ottaviano, L. Patterning at the nanoscale: Atomic force microscopy and extreme ultraviolet interference lithography. Mater. Sci. Eng. B 2009, 165, 227-230.

181. Ramanan, V.; Nelson, E.; Brzezinski, A.; Braun, P.V.; Wiltzius, P. Three dimensional silicon-air photonic crystals with controlled defects using interference lithography. Appl. Phys. Lett. 2008, 92, 173304. 
182. Lai, N.D.; Lin, J.H.; Liang, W.P.; Hsu, C.C.; Lin, C.H. Precisely introducing defects into periodic structures by using a double-step laser scanning technique. Appl. Opt. 2006, 45, 5777-5782.

183. Scrimgeour, J.; Sharp, D.N.; Blanford, C.F.; Roche, O.M.; Denning, R.G.; Turberfield, A.J. Three-dimensional optical lithography for photonic microstructures. Adv. Mater. 2006, 18, 1557-1560.

184. Rumpf, R.C.; Johnson, E.G. Fully three-dimensional modeling of the fabrication and behavior of photonic crystals formed by holographic lithography. J. Opt. Soc. Am. A 2004, 21, 1703-1713.

185. Liu, C.H.; Hong, M.H.; Lum, M.C.; Flotow, H.; Ghadessy, F.; Zhang, J.B. Large-area micro/nanostructures fabrication in quartz by laser interference lithography and dry etching. Appl. Phys. A 2010, 101, 237-241.

186. Salaun, M.; Audier, M.; Delyon, F.; Duneau, M. 3-D holographic lithography of organic-inorganic hybrids. Appl. Surf. Sci. 2007, 254, 830-835.

187. Saravanamuttu, K.; Blanford, C.F.; Sharp, D.N.; Dedman, E.R.; Turberfield, A.J.; Denning, R.G. Sol-gel organic-inorganic composites for 3-D holographic lithography of photonic crystals with submicron periodicity. Chem. Mater. 2003, 15, 2301-2304.

188. Gronheid, R.; Leeson, M.J. Extreme ultraviolet interference lithography as applied to photoresist studies. J. Microlith. Microfab. Microsys. 2009, 8, 021205.

189. Liu, Y.J.; Sun, X.W. Electrically tunable two-dimensional holographic photonic crystal fabricated by a single diffractive element. Appl. Phys. Lett. 2006, 89, 171101.

190. Teteris, J. Immersion holography based on amorphous chalcogenide films. J. Mater. Sci. Mater. Electron. 2009, 20, 149-152.

191. Shishido, A.; Diviliansky, I.B.; Khoo, I.C.; Mayer, T.S.; Nishimura, S.; Egan, G.L.; Mallouk, T.E. Direct fabrication of two-dimensional titania arrays using interference photolithography. Appl. Phys. Lett. 2001, 79, 3332-3334.

192. Guo, Z.G.; Qu, S.L.; Han, Y.H.; Liu, S.T. Multi-photon fabrication of two-dimensional periodic structure by three interfered femtosecond laser pulses on the surface of the silica glass. Opt. Commun. 2007, 280, 23-26.

193. Vlcek, M.; Schroeter, S.; Brueckner, S.; Fehling, S.; Fiserova, A. Direct fabrication of surface relief gratings in chalcogenide glasses by excimer laser interference lithography. J. Mater. Sci. Mater. Electron. 2009, 20, 290-293.

194. Duarte, M.; Lasagni, A.; Giovanelli, R.; Narciso, J.; Louis, E.; Mucklich, F. Increasing lubricant film lifetime by grooving periodical patterns using laser interference metallurgy. Adv. Eng. Mater. 2008, 10, 554-558.

195. Lasagni, A.; Peng, S.; Hendricks, J.L.; Shaw, C.M.; Martin, D.C.; Das, S. Direct fabrication of periodic patterns with hierarchical sub-wavelength structures on poly(3,4-ethylene dioxythiophene)poly(styrene sulfonate) thin films using femtosecond laser interference patterning. Appl. Surf. Sci. 2010, 256, 1708-1713.

196. Everitt, H.O. Applications of photonic band gap structures. Opt. Photon. News 1992, 3, 20-23.

197. Parker, G.; Charlton, M. Photonic crystals. Phys. World 2000, 13, 29-34. 
198. Chen, J.Q.; Jiang, W.; Chen, X.N.; Wang, L.; Zhang, S.S.; Chen, R.T. Holographic three-dimensional polymeric photonic crystals operating in the $1550 \mathrm{~nm}$ window. Appl. Phys. Lett. 2007, 90, 093102.

199. Zhong, Y.C.; Zhu, S.A.; Su, H.M.; Wang, H.Z.; Chen, J.M.; Zeng, Z.H.; Chen, Y.L. Photonic crystal with diamondlike structure fabricated by holographic lithography. Appl. Phys. Lett. 2005, 87, 61103-61101.

200. Yang, X.L.; Cai, L.Z.; Liu, Q.; Liu, H.K. Theoretical bandgap modeling of two-dimensional square photonic crystals fabricated by the interference of three noncoplanar laser beams. J. Opt. Soc. Am. B 2004, 21, 1699-1702.

201. Yang, X.L.; Cai, L.Z.; Wang, Y.R. Larger bandgaps of two-dimensional triangular photonic crystals fabricated by holographic lithography can be realized by recording geometry design. Opt. Express 2004, 12, 5850-5856.

202. Xia, D.Y.; Zhang, J.Y.; He, X.; Brueck, S.R.J. Fabrication of three-dimensional photonic crystal structures by interferometric lithography and nanoparticle self-assembly. Appl. Phys. Lett. 2008, 93, 071105.

203. Moon, J.H.; Small, A.; Yi, G.-R.; Lee, S.-K.; Chang, W.-S.; Pine, D.J.; Yang, S.-M. Patterned polymer photonic crystals using soft lithography and holographic lithography. Synth. Met. 2005, 148, 99-102.

204. Chan, T.Y.M.; John, S. Circuits for light in holographically defined photonic-band-gap materials. Phys. Rev. A 2008, 78, 033812.

205. Cho, C.O.; Jeong, J.; Lee, J.; Jeon, H.; Kim, I.; Jang, D.H.; Park, Y.S.; Woo, J.C. Photonic crystal band edge laser array with a holographically generated square-lattice pattern. Appl. Phys. Lett. 2005, 87, 161102.

206. Gauthier, R.C.; Mnaymneh, K. Towards physical implementation of an optical add-drop multiplexer (OADM) based upon properties of 12-fold photonic quasicrystals. Proc. SPIE 2005, 5970, 59700.

207. Geyer, U.; Hauss, J.; Riedel, B.; Gleiss, S.; Lemmer, U.; Gerken, M. Large-scale patterning of indium tin oxide electrodes for guided mode extraction from organic light-emitting diodes. J. Appl. Phys. 2008, 104, 093111.

208. Liang, G.Q.; Mao, W.D.; Pu, Y.Y.; Zou, H.; Wang, H.Z.; Zeng, Z.H. Fabrication of two-dimensional coupled photonic crystal resonator arrays by holographic lithography. Appl. Phys. Lett. 2006, 89, 41902.

209. Lu, Q.-Y.; Zhang, W.; Wang, L.-J.; Liu, J.-Q.; Li, L.; Liu, F.-Q.; Wang, Z.-G. Holographic fabricated photonic-crystal distributed-feedback quantum cascade laser with near-diffractionlimited beam quality. Opt. Express 2009, 17, 18900-18905.

210. Klein, M.W.; Enkrich, C.; Wegener, M.; Linden, S. Second-harmonic generation from magnetic metamaterials. Science 2006, 313, 502-504.

211. Pendry, J.B.; Holden, A.J.; Robbins, D.J.; Stewart, W.J. Magnetism from conductors and enhanced nonlinear phenomena. IEEE T. Microw. Theory 1999, 47, 2075-2084.

212. Pendry, J.B.; Smith, D.R. The quest for the superlens. Sci. Amer. 2006, 295, 60-67.

213. Schurig, D.; Mock, J.J.; Justice, B.J.; Cummer, S.A.; Pendry, J.B.; Starr, A.F.; Smith, D.R. Metamaterial electromagnetic cloak at microwave frequencies. Science 2006, 314, 977-980. 
214. Veselago, V.G. The electrodynamics of substances simultaneously negative values of $\varepsilon$ and $\mu$. Sov. Phys. Usp. 1968, 10, 509-514.

215. Caloz, C.; Itoh, T. Metamaterials for high-frequency electronics. Proc. IEEE 2005, 93, 1744-1751.

216. Grzegorczyk, T.M.; Moss, C.D.; Lu, J.; Chen, X.; Pacheco, J., Jr,; Kong, J.A. Properties of left-handed metamaterials: Transmission, backward phase, negative refraction, and focusing. IEEE Trans. Microw. Theory 2005, 53, 2956-2966.

217. Hardy, W.N.; Whitehead, L.A. Split-ring resonator for use in magnetic resonance from 200-2000 MHz. Rev. Sci. Instrum. 1981, 52, 213-216.

218. Dolling, G.; Enkrich, C.; Wegener, M.; Soukoulis, C.M.; Linden, S. Simultaneous negative phase and group velocity of light in a metamaterial. Science 2006, 312, 892-894.

219. Linden, S.; Enkrich, C.; Dolling, G.; Klein, M.W.; Zhou, J.; Koschny, T.; Soukoulis, C.M.; Burger, S.; Schmidt, F.; Wegener, M. Photonic metamaterials: Magnetism at optical frequencies. IEEE J. Sel. Top. Quantum Electron. 2006, 12, 1097-1105.

220. Zheludev, N.I. The road ahead for metamaterials. Science 2010, 328, 582-583.

221. Liang, G.Q.; Mao, W.D.; Zou, H.; Chen, B.C.; Cao, J.F.; Pu, Y.Y.; Wen, X.W.; Wang, H.Z. Holographic formation of large area split-ring arrays for magnetic metamaterials. J. Mod. Opt. 2008, 55, 1463-1472.

222. Feth, N.; Enkrich, C.; Wegener, M.; Linden, S. Large-area magnetic metamaterials via compact interference lithography. Opt. Express 2007, 15, 501-507.

223. Li, Q.; Wang, G.P. Tunable photonic metamaterials in the near infrared frequencies. Opt. Express 2010, 18, 14123-14128.

224. Yang, Y.; Li, Q.Z.; Wang, G.P. Design and fabrication of diverse metamaterial structures by holographic lithography. Opt. Express 2008, 16, 11275-11280.

225. Tamma, V.A.; Lee, J.-H.; Wu, Q.; Park, W. Visible frequency magnetic activity in silver nanocluster metamaterial. Appl. Opt. 2010, 49, A11-A17.

226. Kikuta, H.; Toyota, H.; Yu, W. Optical elements with subwavelength structured surfaces. Opt. Rev. 2003, 10, 63-73.

227. Gaylord, T.K.; Baird, W.E.; Moharam, M.G. Zero-reflectivity high spatial-frequency rectangular-groove dielectric surface-relief gratings. Appl. Opt. 1986, 25, 4562-4567.

228. Sawaki, D.; Amako, J. Deep-UV laser-based nano-patterning with holographic techniques. Proc. SPIE 2007, 6459, 64590F.

229. Amako, J.; Sawaki, D.; Fujii, E. High-efficiency diffractive beam splitters surface-structured on submicrometer scale using deep-UV interference lithography. Appl. Opt. 2009, 48, 5105-5113.

230. Yu, J.S.; Song, Y.M.; Leem, J.W.; Lee, Y.T. Subwavelength antireflection structures and their device applications. Proc. SPIE 2010, 7608, 760812.

231. Chen, Y.-P.; Chiu, H.-C.; Chen, G.-Y.; Chiang, C.-H.; Tseng, C.-T.; Lee, C.-H.; Wang, L.A. Fabrication and measurement of large-area sub-wavelength structures with broadband and wide-angle antireflection effect. Microelectron. Eng. 2010, 87, 1323-1327.

232. Gaylord, T.K.; Glytsis, E.N.; Moharam, M.G. Zero-reflectivity homogeneous layers and high spatial-frequency surface-relief gratings on lossy materials. Appl. Opt. 1987, 26, 3123-3135.

233. Hartman, N.F.; Gaylord, T.K. Antireflection gold surface-relief gratings: Experimental characteristics. Appl. Opt. 1988, 27, 3738-3743. 
234. Clapham, P.B.; Hutley, M.C. Reduction of lens reflection by the 'moth eye' principle. Nature 1973, 244, 281-282.

235. MacLeod, B.; Sonek, G. Motheye surfaces reflect little light. Laser Focus World 1999, 35, 109-114.

236. Nakano, H.; Morita, H.; Washida, H.; Kato, T.; Hayashi, S.; Onoe, A. Low cost and high performance antireflective coatings for solar cells. Opt. Engr. 1985, 24, $207-212$.

237. Sopori, B.L.; Pryor, R.A. Design of antireflection coatings for textured silicon solar cells. Solar Cells 1983, 8, 249-261.

238. Song, Y.M.; Yu, J.S.; Lee, Y.T. Antireflective submicrometer gratings on thin-film silicon solar cells for light-absorption enhancement. Opt. Lett. 2010, 35, 276-278.

239. Zhuang, L.; Schablitsky, S.; Shi, R.C.; Chou, S.Y. Fabrication and performance of thin amorpnous Si subwavelength transmission grating for controlling vertical cavity surface emitting laser polarization. J. Vac. Sci. Technol. B 1996, 14, 4055-4057.

240. Minseung, A.; Heilmann, R.K.; Schattenburg, M.L. Fabrication of ultrahigh aspect ratio freestanding gratings on silicon-on-insulator wafers. J. Vac. Sci. Technol. B 2007, 25, 2593-2597.

241. Brundrett, D.L.; Glytsis, E.N.; Gaylord, T.K. Subwavelength transmission grating retarders for use at 10.6um. Appl. Opt. 1996, 35, 6195-6202.

242. Magnusson, R.; Wang, S.S. New principle for optical filters. Appl. Phys. Lett. 1992, 61, 1022-1024.

243. Magnusson, R.; Wang, S.S. Transmission bandpass guided-mode resonance filters. Appl. Opt. 1995, 34, 8106-8109.

244. Tibuleac, S.; Magnusson, R. Reflection and transmission guided-mode resonance filters. J. Opt. Soc. Am. A 1997, 14, 1617-1626.

245. Shokooh-Saremi, M.; Magnusson, R. Leaky-mode resonant reflectors with extreme bandwidths. Opt. Lett. 2010, 35, 1121-1123.

246. Colgan, M.J.; Brett, M.J. Field emission from carbon and silicon films with pillar microstructure. Thin Solid Films 2001, 389, 1-4.

247. Xu, N.S.; Huq, S.E. Novel cold cathode materials and applications. Mater. Sci. Eng. R 2005, 48, 47-189.

248. Spallas, J.P.; Hawryluk, A.M.; Kania, D.R. Field emitter array mask patterning using laser interference lithography. J. Vac. Sci. Technol. B 1995, 13, 1973-1978.

249. Dawood, M.K.; Liew, T.H.; Lianto, P.; Hong, M.H.; Tripathy, S.; Thong, J.T.L.; Choi, W.K. Interference lithographically defined and catalytically etched, large-area silicon nanocones from nanowires. Nanotechnology 2010, 21, 205305.

250. Yun, J.; Wang, R.; Choi, W.K.; Thong, J.T.L.; Thompson, C.V.; Mei, Z.; Foo, Y.L.; Hong, M.H. Field emission from a large area of vertically-aligned carbon nanofibers with nanoscale tips and controlled spatial geometry. Carbon 2010, 48, 1362-1368.

251. Li, H.; Luo, X.; Du, C.; Chen, X.; Fu, Y. Ag dots array fabricated using laser interference technique for biosensing. Sens. Actuat. B 2008, 134, 940-944.

252. Sahoo, P.K.; Vogelsang, K.; Schift, H.; Solak, H.H. Surface plasmon resonance in near-field coupled gold cylinder arrays fabricated by EUV-interference lithography and hot embossing. Appl. Surf. Sci. 2009, 256, 431-434. 
253. Liu, C.H.; Hong, M.H.; Cheung, H.W.; Zhang, F.; Huang, Z.Q.; Tan, L..; Hor, T.S.A. Bimetallic structure fabricated by laser interference lithography for tuning surface plasmon resonance. Opt. Express 2008, 16, 10701-10709.

254. Menezes, J.W.; Braga, E.S.; Cescato, L. Photonic crystals and plasmonic structures recorded by multi-exposure of holographic patterns. Proc. SPIE 2009, 7358, 73580K.

255. Daniel, C. Biomimetic structures for mechanical applications by interfering laser beams: more than solely holographic gratings. J. Mater. Res. 2006, 21, 2098-2105.

256. Wu, D.; Chen, Q.-D.; Yao, J.; Guan, Y.-C.; Wang, J.-N.; Niu, L.-G.; Fang, H.-H.; Sun, H.-B. A simple strategy to realize biomimetic surfaces with controlled anisotropic wetting. Appl. Phys. Lett. 2010, 96, 053704.

257. Yang, Y.-L.; Hsu, C.-C.; Chang, T.-L.; Kuo, L.-S.; Chen, P.-H. Study on wetting properties of periodical nanopatterns by a combinative technique of photolithography and laser interference lithography. Appl. Surf. Sci. 2010, 256, 3683-3687.

258. Zhang, Z.; Wang, X.; Liu, Y.; Guo, Y.; Hong, Y.; Xu, X.; Fu, S.; Xu, P.; Wang, J.; Cai, J. Fabrication of CoFe nanostructures by holographic lithography. Proc. SPIE 2008, 6831, 68311B.

259. Murillo, R.; van Wolferen, H.A.; Abelmann, L.; Lodder, J. C. Fabrication of patterned magnetic nanodots by laser interference lithography. Microelectron. Eng. 2005, 78-79, 260-265.

260. Rosa, W.O.; Knobel, M.; Cescato, L.; Gobbi, A.L.; Vazquez, M. Experimental magnetic study and evidence of the exchange bias effect in unidimensional Co arrays produced by interference lithography. Solid State Commun. 2007, 142, 228-231.

261. Leufke, P.M.; Riedel, S.; Lee, M.-S.; Li, J.; Rohrmann, H.; Eimuller, T.; Leiderer, P.; Boneberg, J.; Schatz, G.; Albrecht, M. Two different coercivity lattices in Co/Pd multilayers generated by single-pulse direct laser interference lithography. J. Appl. Phys. 2009, 105, 113915.

262. Sinclair, G.; Jordan, P.; Courtial, J.; Padgett, M.; Cooper, J.; Laczik, Z.J. Assembly of 3-dimensional structures using programmable holographic optical tweezers. Opt. Express 2004, $12,5475-5480$.

263. Leach, J.; Sinclair, G.; Jordan, P.; Courtial, J.; Padgett, M.J.; Cooper, J.; Laczik, Z.J. 3D manipulation of particles into crystal structures using holographic optical tweezers. Opt. Express 2004, 12, 220-226.

264. Horner, F.; Woerdemann, M.; Muller, S.; Maier, B.; Denz, C. Full 3D translational and rotational optical control of multiple rod-shaped bacteria. J. Biophotonics 2010, 3, 468-475.

265. Woerdemann, M.; Glasener, S.; Horner, F.; Devaux, A.; De Cola, L.D.; Denz, C. Dynamic and reversible organization of zeolite $\mathrm{L}$ crystals induced by holographic optical tweezers. Adv. Mater. 2010, 22, 4176-4179.

266. Chiou, A.E.; Wang, W.; Sonek, G.J.; Hong, J.; Berns, M.W. Interferometric optical tweezers. Opt. Commun. 1997, 133, 7-10.

267. MacDonald, M.P.; Volke-Sepulveda, K.; Paterson, L.; Arlt, J.; Sibbett, W.; Dholakia, K. Revolving interference patterns for the rotation of optically trapped particles. Opt. Commun. 2002, 201, 21-28.

268. MacDonald, M.P.; Paterson, L.; Volke-Sepulveda, K.; Arlt, J.; Sibbett, W.; Dholakia, K. Creation and manipulation of three-dimensional optically trapped structures. Science 2002, 296, 1101-1103. 
269. Casaburi, A.; Pesce, G.; Zemánek, P.; Sasso, A. Two- and three-beam interferometric optical tweezers. Opt. Commun. 2005, 251, 393-404.

270. Jonas, A.; Zemanek, P. Light at work: The use of optical forces for particle manipulation, sorting, and analysis. Electrophoresis 2008, 29, 4813-4851.

271. MacDonald, M.P.; Paterson, L.; Sibbett, W.; Dholakia, K.; Bryant, P.E. Trapping and manipulation of low-index particles in a two-dimensional interferometric optical trap. Opt. Lett. 2001, 26, 863-865.

272. Schonbrun, E.; Piestun, R.; Jordan, P.; Cooper, J.; Wulff, K.D.; Courtial, J.; Padgett, M. 3D interferometric optical tweezers using a single spatial light modulator. Opt. Express 2005, 13, 3777-3786.

273. Jakl, P.; Cizmar, T.; Sery, M.; Zemanek, P. Static optical sorting in a laser interference field. Appl. Phys. Lett. 2008, 92, 161110-161113.

274. Wieringa, P.A.; Wiertz, R.W.F.; de Weerd, E.L.; Rutten, W.L.C. In vitro verification of a 3-D regenerative neural interface design: Examination of neurite growth and electrical properties within a bifurcating microchannel structure. Proc. IEEE 2010, 98, 389-397.

275. Lutolf, M.P.; Weber, F.E.; Schmoekel, H.G.; Schense, J.C.; Kohler, T.; Muller, R.; Hibbell, J.A. Repair of bone defects using synthetic mimetics of collagenous extracellular matrices. Nature Biotechnol. 2003, 21, 513-518.

276. Capadona, J.R.; Petrie, T.A.; Fears, K.P.; Latour, R.A.; Collard, D.M.; Garcia, A.J. Surface-nucleated assembly of fibrillar extracellular matrices. Adv. Mater. 2005, 17, 2604-2608.

277. Gallant, N.D.; Capadona, J.R.; Frazier, A.B.; Collard, D.M.; Garcia, A.J. Micropatterned surfaces to engineer focal adhesions for analysis of cell adhesion stregthening. Langmuir 2002, $18,5579-5584$.

278. Koch, W.H. Technology platforms for pharmacogenomic diagnostic assays. Nat. Rev. Drug Discov. 2004, 3, 749-761.

279. Sniadecki, N.J.; Desai, R.A.; Ruiz, S.A.; Chen, C.S. Nanotechnology for cell-substrate interactions. Ann. Biomed. Eng. 2006, 34, 59-74.

280. Zhao, G.; Zinger, O.; Schwartz, Z.; Wieland, M.; Landolt, D.; Boyan, B.D. Osteoblast-like cells are sensitive to submicron-scale surface structure. Clin. Oral Implan. Res. 2006, 17, 258-264.

281. Zoller, F.A.; Padeste, C.; Ekinci, Y.; Solak, H.H.; Engel, A. Nanostructured substrates for high density protein arrays. Microelectron. Eng. 2008, 85, 1370-1374.

282. Hedberg-Dirk, E.L.; Martinez, U.A. Large-scale protein arrays generated with interferometric lithography for spatial control of cell-material interactions. J. Nanomater. 2010, 176750.

283. Akhavan, O.; Abdolahad, M.; Asadi, R. Storage of Ag nanoparticles in pore-arrays of SU-8 matrix for antibacterial applications. J. Phys. D Appl. Phys. 2009, 42.

(C) 2011 by the authors; licensee MDPI, Basel, Switzerland. This article is an open access article distributed under the terms and conditions of the Creative Commons Attribution license (http://creativecommons.org/licenses/by/3.0/). 